\title{
RESONANCES FOR 1D STARK OPERATORS
}

\author{
EVGENY L. KOROTYAEV
}

\begin{abstract}
We consider the Stark operator perturbed by a compactly supported potential (of a certain class) on the real line. We prove the following results: (a) upper and lower bounds on the number of resonances in complex discs with large radii, (b) the trace formula in terms of resonances only, (c) all resonances determine the potential uniquely.
\end{abstract}

Dedicated to the memory of Professor Viktor Havin, (St.Petersburg, 1933-2015)

\section{INTRODUCTION AND MAIN RESULTS}

1.1. Introduction. We consider the operator $H=H_{0}+V$ acting on $L^{2}(\mathbb{R})$, where the unperturbed operator $H_{0}$ is the Stark operator given by

$$
H_{0}=-\frac{d^{2}}{d x^{2}}+x
$$

Here $x$ is an external electric field and the potential $V=V(x), x \in \mathbb{R}$ is real and satisfies

Condition V. The potential $V \in L_{\text {real }}^{2}(\mathbb{R})$ and $\operatorname{supp} V \subset[0, \gamma]$ for some $\gamma>0$.

Our main results devote to the asymptotics of the number of resonances in large discs and an inverse problem in terms of resonances (all resonances determine the potential uniquely). Under Condition $\mathrm{V}$ the operator $V\left(H_{0}-i\right)^{-1}$ is compact (see Lemma 2.1). Then the operators $H_{0}$ and $H$ are self-adjoint on the same domain and $C_{0}^{\infty}(\mathbb{R})$ is a core for both $H_{0}$ and $H$. The spectrum of both $H_{0}$ and $H$ is purely absolutely continuous and covers the real line $\mathbb{R}$ (see Avron-Herbst [3] and Herbst [14]).

The Stark effect is the shifting and splitting of spectral lines of atoms and molecules due to presence of an external electric field. The effect is named after Stark, who discovered it in 1913. The Stark effect has been of marginal benefit in the analysis of atomic spectra, but has been a major tool for molecular rotational spectra. The perturbation theory for the Stark effect has some problems. In absence of an electric field, states of atoms and molecules are square-integrable. In the presence of an electric field, states of atoms and molecules are not square-integrable and they becomes resonances of finite width. For weak fields low lying states can be regarded as bound, but for all other cases we need to calculate resonances and the corresponding states, which are not square-integrable.

It is well known that the wave operators $W_{ \pm}$for the pair $H_{0}, H$ given by

$$
W_{ \pm}=s-\lim e^{i t H} e^{-i t H_{0}} \quad \text { as } \quad t \rightarrow \pm \infty,
$$

exist and are unitary (even under much less restrictive assumptions on the potential than considered here, see [3, 14]). Thus the scattering operator $S=W_{+}^{*} W_{-}$is unitary. The

Date: July 4, 2021.

1991 Mathematics Subject Classification. 34F15( 47E05).

Key words and phrases. Stark operators, resonances, trace formula . 
operators $H_{0}$ and $S$ commute and thus are simultaneously diagonalizable:

$$
L^{2}(\mathbb{R})=\int_{\mathbb{R}}^{\oplus} \mathscr{H}_{\lambda} d \lambda, \quad H_{0}=\int_{\mathbb{R}}^{\oplus} \lambda I_{\lambda} d \lambda, \quad S=\int_{\mathbb{R}}^{\oplus} S(\lambda) d \lambda ;
$$

here $I_{\lambda}$ is the identity in the fiber space $\mathscr{H}_{\lambda}=\mathbb{C}$ and $S(\lambda)$ is the scattering matrix (which is a scalar function in $\lambda \in \mathbb{R}$ for our case) for the pair $H_{0}, H$ (see Yajima [48]).

1.2. Determinants. The main objects studied in the present paper are the resonances of $H$ and the scattering matrix $S(\lambda)=e^{-2 \pi i \phi_{s c}(\lambda)}, \lambda \in \mathbb{R}$ for the pair $H_{0}, H$, where $\phi_{s c}(\lambda)$ is the scattering phase (or the spectral shift function in the terminology associated with the trace formula). In order to study resonances we chose an approach where a central role is played by the Fredholm determinant. More precisely, we set

$$
\begin{aligned}
& V=|V|^{\frac{1}{2}} V^{\frac{1}{2}}, \quad V^{\frac{1}{2}}=|V|^{\frac{1}{2}} \operatorname{sign} V, \\
& R_{0}(\lambda)=\left(H_{0}-\lambda\right)^{-1}, \quad Y_{0}(\lambda)=|V|^{\frac{1}{2}} R_{0}(\lambda) V^{\frac{1}{2}}, \quad \lambda \in \mathbb{C}_{ \pm} .
\end{aligned}
$$

Here $\mathbb{C}_{ \pm}=\{\lambda \in \mathbb{C}: \pm \operatorname{Im} \lambda>0\}$ denote the upper and lower half plane and $\lambda$ is a spectral parameter. We shortly describe standard properties of the operator-valued function $Y_{0}$, which we will prove in Section 2. We shall show that each operator $Y_{0}(\lambda), \operatorname{Im} \lambda \neq 0$, is trace class and thus we can define the determinant:

$$
D_{ \pm}(\lambda)=\operatorname{det}\left(I+Y_{0}(\lambda)\right), \quad \lambda \in \mathbb{C}_{ \pm} .
$$

Moreover, we show that the function $D_{ \pm}(\lambda), \lambda \in \mathbb{C}_{ \pm}$is analytic in $\mathbb{C}_{ \pm}$, continuous up to the real line and $D_{ \pm}(\lambda) \neq 0$ for all $\lambda \in \overline{\mathbb{C}}_{ \pm}$. Furthermore, the function $D_{ \pm}$satisfies

$$
D_{ \pm}(\lambda)=1+O\left(\lambda^{-a}\right) \quad \text { as } \quad|\lambda| \rightarrow \infty, \quad \lambda \in \overline{\mathbb{C}}_{ \pm},
$$

for any fixed $a \in\left(0, \frac{1}{2}\right)$, uniformly with respect to $\arg \lambda \in[0, \pm \pi]$. Thus we can define the branch $\log D_{ \pm}(\lambda), \lambda \in \mathbb{C}_{ \pm}$by $\log D_{ \pm}(\lambda)=O\left(\lambda^{-a}\right)$ as $|\lambda| \rightarrow \infty, \lambda \in \mathbb{C}_{ \pm}$. For each $\lambda \in \mathbb{R}$ the following identities hold true:

$$
S(\lambda)=\frac{\bar{D}_{+}(\lambda+i 0)}{D_{+}(\lambda+i 0)}=\frac{D_{-}(\lambda-i 0)}{D_{+}(\lambda+i 0)}=e^{-2 \pi i \phi_{s c}(\lambda)},
$$

where $\phi_{s c}=\frac{1}{\pi} \arg D_{+}(\lambda+i 0)$ is the scattering phase. Thus the standard arguments give that the function $S(\lambda)$, defined by (1.2), is continuous in $\lambda \in \mathbb{R}$. The basic properties of determinants (see below (2.5),$(2.2)$ ) give the identity

$$
\bar{D}_{+}(\lambda)=\operatorname{det}\left(I+Y_{0}(\lambda)^{*}\right)=\operatorname{det}\left(I+Y_{0}(\bar{\lambda})\right)=D_{-}(\bar{\lambda}) \quad \forall \quad \lambda \in \mathbb{C}_{+} .
$$

Due to this identity it is enough to consider $D_{+}$or $D_{-}$. Our first preliminary theorem describes the Fredholm determinant $D_{+}(\lambda)$ and its asymptotics at high energy.

Condition C. The potential $V$ satisfies Condition $V$ and the restriction of $V$ on the interval $(0, \gamma)$ is absolutely continuous.

Theorem 1.1. Let $V$ satisfy Condition $\mathrm{C}$ and and let $\tau<1$. Then the function $\log D_{+}(\lambda)$ is analytic in $\mathbb{C}_{+}$, continuous up to the real line and satisfies

$$
\log D_{+}(\lambda)=\frac{i V_{0}}{2 \sqrt{\lambda}}+\frac{O(1)}{\lambda^{\tau}} \quad \text { as }|\lambda| \rightarrow \infty, \lambda \in \overline{\mathbb{C}}_{+},
$$


where $V_{0}=\int_{\mathbb{R}} V(x) d x$, uniformly with respect to $\arg \lambda \in[0, \pi]$. In particular,

$$
\begin{array}{rlrlrl}
\phi_{s c}(\lambda) & =\frac{V_{0}}{2 \pi \sqrt{\lambda}}+\frac{O(1)}{\lambda^{\tau}} & \text { as } & \lambda \rightarrow+\infty, \\
\phi_{s c}(\lambda) & =O\left(1 / \lambda^{\tau}\right) & \text { as } & \lambda & \rightarrow-\infty .
\end{array}
$$

Remark. 1) Under our assumptions on $V$ the proof of the asymptotic expansion (1.8) is a bit technical. If $V$ is, e.g. in the Schwartz class, then the expansion becomes much easier and higher order terms can be derived as well (see [20]), similar to the 3-dim case in [31].

1.3. Resonances. In order to describe resonances we recall the definition of order and type. Definition. The entire function $f$ is of order $\beta$ if

$$
\limsup _{r \rightarrow \infty} \frac{\log \log M(r)}{\log r}=\beta,
$$

where $M(r)=\sup _{|z|=r}|f(z)|$. The function $f$ of positive order $\beta>0$ is of type $a \geqslant 0$ if

$$
\limsup _{r \rightarrow \infty} \frac{\log M(r)}{r^{\beta}}=a .
$$

Under Condition $\mathrm{V}$ we will obtain an analytic continuation of $D_{+}(\lambda), \lambda \in \mathbb{C}_{+}$to the entire complex plane and information on its zeros and obtain upper bounds on the number of resonances of the operator $H$. We denote by $\left(\lambda_{n}\right)_{1}^{\infty}$ the sequence of zeros in $\mathbb{C}_{-}$of $D_{+}$(counting multiplicities), arranged such that

$$
0<\left|\lambda_{1}\right| \leqslant\left|\lambda_{2}\right| \leqslant\left|\lambda_{2}\right| \leqslant \ldots
$$

By definition, a zero $\lambda_{n} \in \mathbb{C}_{-}$of $D_{+}$is called a resonance. The multiplicity of the resonance is the multiplicity of the corresponding zero of $D_{+}$. In order to obtain lower bounds on the number of resonances we assume that the potential $V$ satisfies Condition $\mathrm{C}$ with $V \neq 0$. More precisely

Theorem 1.2. Let $V$ satisfy Condition $V$. Then $D_{ \pm}(\lambda), \lambda \in \mathbb{C}_{ \pm}$has an analytic extension into the whole complex plane and satisfies

$$
\left|D_{ \pm}(\lambda)\right| \leqslant C_{0} e^{\frac{4}{3}|\lambda|^{\frac{3}{2}}} \quad \forall \lambda \in \mathbb{C},
$$

for some constant $C_{0}$. Furthermore, by (1.6), the $S$-matrix $S(\lambda), \lambda \in \mathbb{R}$ has an analytic extension into the whole upper half plane $\mathbb{C}_{+}$and a meromorphic extension into the whole lower half plane $\mathbb{C}_{-}$. The zeros of $S(\lambda), \lambda \in \mathbb{C}_{+}$coincide with the zeros of $D_{-}$and the poles of $S(\lambda), \lambda \in \mathbb{C}_{-}$are precisely the zeros of $D_{+}$. Let, in addition, $V$ satisfy Condition $\mathrm{C}$ and $V(0) \neq 0$. Then $D_{ \pm}$is an entire function of order $\frac{3}{2}$ and type $\frac{4}{3}$.

Remark. 1) From (1.13) we deduce that $D_{+}(\lambda)$ has the Hadamar factorization:

$$
D_{+}(\lambda)=D_{+}(0) e^{p \lambda} \lim _{r \rightarrow+\infty} \prod_{\left|\lambda_{n}\right| \leqslant r}\left(1-\frac{\lambda}{\lambda_{n}}\right) e^{\frac{\lambda}{\lambda_{n}}}, \quad \lambda \in \mathbb{C},
$$

uniformly on any compact subset of $\mathbb{C}$, where the constant $p$ satisfies

$$
p=\frac{D_{+}^{\prime}(0)}{D_{+}(0)}, \quad \operatorname{Im} p=\pi \phi_{s c}^{\prime}(0),
$$


with $\phi_{s c}(\cdot)$ defined in (1.6).

2) By (1.14), the operator $H$ has an infinite number of resonances.

3) Due to (1.6) the resonances are the zeros $\lambda_{n} \in \mathbb{C}_{-}, n \geqslant 1$ of $D_{+}$(and the poles of $S(\lambda)$ with the same multiplicity) in $\mathbb{C}_{-}$labeled according to (1.12). The zeros of the S-matrix $S(\lambda)$ are the zeros of $D_{-}$in $\mathbb{C}_{-}$given by $\bar{\lambda}_{n} \in \mathbb{C}_{-}, n \geqslant 1$.

Denote by $\mathcal{N}(r, f)$ the number of zeros (counted according to multiplicity) of $f$ having modulus $\leqslant r$. The Lindelöf Theorem jointly with Theorem 1.2 applied to the Fredholm determinant of the perturbed Stark operator gives

Corollary 1.3. Let $V$ satisfy Condition V. Then the entire function $D_{+}$satisfies

$$
\mathcal{N}\left(r, D_{+}\right) \leqslant C_{1} r^{\frac{3}{2}}
$$

for $r>0$ sufficiently large and for some positive constant $C_{1}$.

Let in addition $V$ satisfy Condition $\mathrm{C}$ and $V(0) \neq 0$. Then there is a sequence of positive numbers $r_{j}, j \in \mathbb{N}$, tending to $\infty$ and a positive constant $C_{0}>0$ such that

$$
\mathcal{N}\left(r_{j}, D_{+}\right) \geqslant C_{0} r_{j}^{\frac{3}{2}} \quad \forall j \in \mathbb{N} .
$$

Remark. 1) We emphasize that the Hadamard factorization of $D_{+}(\lambda)$ in (1.14) crucially depends on determining its order and type (which are equal to the order and type of the squared Airy function, which gives the generalized eigenfunctions for the unperturbed Stark operator). In structure the Hadamard factorization looks similar to the factorization for the Schrödinger operator $-\Delta+V$ in $\mathbb{R}^{3}$, see e.g. [50, 46], 4]. In fact, this is closely connected to counting the number of resonances, by a result of Lindelöf (see [36]), which is contained in Boas's book, see p.25 in [5] and in Section 5.

Thus we see that the perturbed Stark operator $H$ on the real line has much more resonances than the corresponding Schrödinger operator on the real line. In fact, the number of resonances (i.e., $\mathcal{N}\left(r, D_{+}\right) \leqslant C r^{\frac{3}{2}}$ in the disc $\left\{\left|\lambda_{n}\right| \leqslant r\right\}$ ) of the perturbed Stark operator $H$ on the real line corresponds to the one for the Schrödinger operator on $\mathbb{R}^{3}$. Recall that for the Schrödinger operator on $\mathbb{R}^{3}$ the number $\mathcal{N}_{\rho}$ of resonances $\lambda_{n}$ in the $\operatorname{disc}\left\{\left|\lambda_{n}\right| \leqslant \rho^{2}\right\}$ has the bound $\mathcal{N}_{\rho} \leqslant C \rho^{3}=C r^{\frac{3}{2}}$ at $r=\rho^{2}$. This explains the similarity in the Hadamard factorization.

Our next corollary concerns the trace formula in terms of resonances. So far, trace formulas for one-dimensional Schrödinger operators in terms of resonances have only been determined in [28]. Here we also follow the approach in [28].

Corollary 1.4. Let $V$ satisfy Condition $\mathrm{V}$ and let $R(\lambda)=(H-\lambda)^{-1}, \operatorname{Im} \lambda \neq 0$. Then the following identity (the trace formula) holds true:

$$
\operatorname{Tr}\left(R_{0}(\lambda)-R(\lambda)\right)=p+\sum_{n \geqslant 1} \frac{\lambda}{\lambda_{n}\left(\lambda-\lambda_{n}\right)},
$$

where the series converges absolutely and uniformly on any compact set of $\mathbb{C} \backslash\left\{\lambda_{n}, n \geqslant 1\right\}$.

Remark. We discuss trace formulas in Section 5 and we will show the following identity:

$$
\phi_{s c}^{\prime}(\lambda)=\phi_{s c}^{\prime}(0)+\frac{\lambda}{\pi} \operatorname{Im} \sum_{n \geqslant 1} \frac{1}{\lambda_{n}\left(\lambda-\lambda_{n}\right)} \quad \forall \lambda \in \mathbb{R},
$$

uniformly on any compact subset of $\mathbb{R}$. Note that the identity (1.19) is a Breit-Wigner type formula for resonances (see p. 53 of [41]). 
We discuss now inverse resonance problems. We show that all resonances determine the potential uniquely. It is a first result about inverse resonance problems for perturbed Stark operators.

Theorem 1.5. Let the perturbed Stark operators $H_{j}=H_{0}+V_{j}, j=1,2$ act on $L^{2}(\mathbb{R})$ and let each potential $V_{j}, j=1,2$ satisfy Condition $\mathrm{V}$. Assume that $H_{1}$ and $H_{2}$ have the same resonances. Then $V_{1}=V_{2}$.

Remark. In the case of Schrödinger operator with a compactly supported potential on the half-line all resonances determine the potential [28]. In the case of the real line all resonances do not determine the potential [29].

1.4. Brief overview. Concerning previous results on resonances, we recall that from a physicists point of view, they were first studied by Regge [42. Since then, properties of resonances have been the object of intense study and we refer to [46] for the mathematical approach in the multi-dimensional case and references given there.

A lot of papers are devoted to resonances of the one-dimensional Schrödinger operator, see Froese [13, Korotyaev 28], Simon [4], Zworski [50] and references given there. We recall that Zworski [50] obtained the first results about the asymptotic distribution of resonances for the Schrödinger operator with compactly supported potentials on the real line (this result is sharper than Corollary 1.3 in the present paper). Inverse problems (characterization, recovering, uniqueness) in terms of resonances were solved by Korotyaev for a Schrödinger operator with a compactly supported potential on the real line [29] and the half-line [28], see also Zworski [51, Brown-Knowles-Weikard [6] concerning the uniqueness.

Next, we mention some results for one-dimensional perturbed Stark operators. The onedimensional scattering theory was considered by Rejto-Sinha [43], Jensen [22], Liu [38]. The one-dimensional inverse scattering problem is studied by Calogero-Degasperis [7], GraffiHarrell [18], Kachalov-Kurylev [24], Kristensson [33], Lin-Qian-Zhang [37]. There are a lot of results about the resonances of the one-dimensional perturbed Stark operator, where the dilation analyticity techniques are used, see e.g., [15], [23] and [8] and references therein. Note that compactly supported potentials are not treated in these papers.

We mention also interesting results about resonances for one-dimensional Stark-Wannier operators $-\frac{d^{2}}{d x^{2}}+\varepsilon x+V_{\pi}$, where the constant $\varepsilon>0$ is the electric field strength and $V_{\pi}$ is the real periodic potential: Agler-Froese [1], Grecchi-Sacchetti [19], Herbst-Howland [16], Jensen [21]. In the case $\varepsilon=0$, the resonances for one-dimensional operators $-\frac{d^{2}}{d x^{2}}+V_{\pi}+V$, where $V$ is a compactly supported potential were considered by Firsova [12, Korotyaev [30], Korotyaev-Schmidt [32].

1.5. Plan of the paper. In Section 2 we recall well known results on the spectral representation of the Stark operator in a form useful for our approach and obtain basic estimates on $Y_{0}(\lambda)$, using Privalov's Lemma. Section 3 contains the stationary representation of the scattering matrix and the proof of Theorem 1.1. Section 4 establishes the analytic continuation of $D_{ \pm}(\lambda), \lambda \in \mathbb{C}_{ \pm}$and the meromorphic continuation of $S(\lambda)$ and gives the crucial estimates on order and type leading to Corollary 1.3. In Section 5 we prove Theorem 1.2 and Theorem 1.4. The Appendix contains technical estimates needed in the proof of Lemma 2.4 which is crucial to obtain the asymptotic expansion (1.8). 


\section{Unperturbed Stark operators}

2.1. The well-known facts. We denote by $C$ various possibly different constants whose values are immaterial in our constructions. By $\mathcal{B}$ and $\mathcal{B}_{\infty}$ we denote the classes of bounded and compact operators, respectively. Let $\mathcal{B}_{1}$ and $\mathcal{B}_{2}$ be the trace and the Hilbert-Schmidt class equipped with the norm $\|\cdot\|_{\mathcal{B}_{1}}$ and $\|\cdot\|_{\mathcal{B}_{2}}$, respectively. We recall some well known facts. Let $A, B \in \mathcal{B}$ and $A B, B A \in \mathcal{B}_{1}$. Then

$$
\begin{aligned}
\operatorname{Tr} A B & =\operatorname{Tr} B A, \\
\operatorname{det}(I+A B) & =\operatorname{det}(I+B A),
\end{aligned}
$$

the mapping $X \rightarrow \operatorname{det}(I+X)$ is continuous on $\mathcal{B}_{1}$,

$$
\begin{aligned}
& |\operatorname{det}(I+X)| \leqslant e^{\|X\|_{\mathcal{B}_{1}}} \\
& \overline{\operatorname{det}(I+X)}=\operatorname{det}\left(I+X^{*}\right) \text {, } \\
& |\operatorname{det}(I+X)-\operatorname{det}(I+Y)| \leqslant\|X-Y\|_{\mathcal{B}_{1}} e^{1+\|X\|_{\mathcal{B}_{1}}+\|Y\|_{\mathcal{B}_{1}}},
\end{aligned}
$$

for all $X, Y \in \mathcal{B}_{1}$, see e.g., Sect. 3. in the book [45]. Let the operator-valued function $\Omega: \mathcal{D} \rightarrow \mathcal{B}_{1}$ be analytic for some domain $\mathcal{D} \subset \mathbb{C}$ and $(I+\Omega(z))^{-1} \in \mathcal{B}$ for any $z \in \mathcal{D}$. Then the function $F(z)=\operatorname{det}(I+\Omega(z))$ satisfies

$$
F^{\prime}(z)=F(z) \operatorname{Tr}(I+\Omega(z))^{-1} \Omega^{\prime}(z), \quad z \in \mathcal{D} .
$$

Recall that the kernels of the operators $(-\Delta-\lambda)^{-1}$ and $e^{i t \Delta}$ on $L^{2}(\mathbb{R})$ have the form

$$
\begin{gathered}
(-\Delta-\lambda)^{-1}(x, y)=\frac{i}{2 \sqrt{\lambda}} e^{i \sqrt{\lambda}|x-y|}, \quad \lambda \in \mathbb{C} \backslash \overline{\mathbb{R}}_{+}, \quad \sqrt{\lambda} \in \mathbb{C}_{+}, \\
\left(e^{i t \Delta}\right)(x, y)=\frac{e^{-i \pi / 4}}{\sqrt{4 \pi t}} e^{i|x-y|^{2} / 4 t}, \quad t \neq 0,
\end{gathered}
$$

$x, y \in \mathbb{R}^{1}$. We need the identities for the Stark operator $H_{0}=-\frac{d^{2}}{d x^{2}}+x$ from [3] given by

$$
e^{-i t H_{0}}=e^{-i t x} e^{i t \Delta} e^{-i \partial t^{2}} e^{-i \frac{t^{3}}{3}}, \quad \forall t \in \mathbb{R} .
$$

where $\partial=-i \frac{d}{d x}, \Delta=\frac{d^{2}}{d x^{2}}$. The free resolvent $R_{0}(\lambda)=\left(H_{0}-\lambda\right)^{-1}$ and its kernel $R_{0}(x, y, \lambda)$ satisfy

$$
\begin{array}{r}
R_{0}(\lambda)=i \int_{0}^{\infty} e^{-i t\left(H_{0}-\lambda\right)} d t=i \int_{0}^{\infty} e^{-i t x} e^{-i \partial t^{2}} e^{i t \Delta} e^{i t \lambda-i \frac{t^{3}}{3}} d t \\
R_{0}(x, y, \lambda)=\frac{e^{i \frac{\pi}{4}}}{\sqrt{4 \pi}} \int_{0}^{\infty} e^{-i t x} e^{\frac{i}{4 t}\left|x-t^{2}-y\right|^{2}} e^{-i \frac{t^{3}}{3}+i t \lambda} \frac{d t}{t^{1 / 2}}
\end{array}
$$

for $x, y \in \mathbb{R}$ and $\lambda \in \mathbb{C}_{+}$. We introduce the resolvent $R(\lambda)$ for $H$ and operators $Y, J$ by

$$
R(\lambda)=(H-\lambda)^{-1}, \quad Y(\lambda)=|V|^{\frac{1}{2}} R(\lambda) V^{\frac{1}{2}}, \quad J(\lambda)=I-Y(\lambda), \quad J_{0}(\lambda)=I+Y_{0}(\lambda),
$$

for $\lambda \in \mathbb{C}_{ \pm}$and recall that $Y_{0}(\lambda)=|V|^{\frac{1}{2}} R_{0}(\lambda) V^{\frac{1}{2}}$. Below we will use the identities

$$
R=R_{0}-R_{0} V R, \quad J(\lambda) J_{0}(\lambda)=I, \quad \lambda \in \mathbb{C}_{ \pm} .
$$


2.2. The spectral representation for $H_{0}$. We will need some facts concerning the spectral decomposition of the Stark operator $H_{0}$, which we denote by

$$
E_{0}(\lambda)=\chi\left(\lambda-H_{0}\right), \quad \lambda \in \mathbb{R}, \quad \text { where } \quad \chi(\lambda)=\left\{\begin{array}{ll}
1 & \lambda \geqslant 0 \\
0 & \lambda<0
\end{array} .\right.
$$

Now we recall formulae for $E_{0}(\lambda)$ due to [3]. Let $\varphi$ be the multiplication operator by the function $\varphi(k)=e^{i k^{3} / 3}, k \in \mathbb{R}$. Then

$$
H_{0}=-\frac{d^{2}}{d x^{2}}+x=\varphi(\partial)^{*}(x) \varphi(\partial), \quad \partial=-i \frac{d}{d x} .
$$

Let $U: f \mapsto \widetilde{f}$ be the unitary transformation on $L^{2}(\mathbb{R})$, which can be defined on $L^{1}(\mathbb{R}) \cap L^{2}(\mathbb{R})$ by the explicit formula

$$
\widetilde{f}(p)=(U f)(p)=\frac{1}{\sqrt{\pi}} \int_{\mathbb{R}} \operatorname{Ai}(x-p) f(x) d x,
$$

where $\operatorname{Ai}(\cdot)$ is the Airy function:

$$
\operatorname{Ai}(z)=\frac{1}{\pi} \int_{0}^{\infty} \cos \left(\frac{t^{3}}{3}+t z\right) d t, \quad \forall z \in \mathbb{R} .
$$

The unitary transformation (2.14) carries $H_{0}$ over into multiplication by $p$ in $L^{2}(\mathbb{R}, d p)$ :

$$
\left(U H_{0} U^{*} \tilde{f}\right)(p)=p \widetilde{f}(p), \quad \tilde{f} \in \mathscr{D}(p) .
$$

Thus, for any $f \in L^{2}(\mathbb{R})$, the quadratic form of $E_{0}(\lambda)$ can be presented as

$$
\left\langle E_{0}(\lambda) f, f\right\rangle=\int_{p<\lambda}|\widetilde{f}(p)|^{2} d p \quad \forall \lambda \in \mathbb{R},
$$

where $\langle\cdot, \cdot\rangle$ is the scalar product in $L^{2}(\mathbb{R})$. Differentiation with respect to $\lambda$ gives

$$
\frac{d}{d \lambda}\left\langle E_{0}(\lambda) f, f\right\rangle=|G(\lambda) f|^{2}, \quad f \in L^{1}(\mathbb{R}) \cap L^{2}(\mathbb{R}),
$$

where $G(\lambda): L^{1}(\mathbb{R}) \cap L^{2}(\mathbb{R}) \rightarrow \mathbb{C}$ is given by

$$
G(\lambda) f:=U(\lambda) f=\frac{1}{\sqrt{\pi}} \int_{\mathbb{R}} \operatorname{Ai}(x-\lambda) f(x) d x \quad \forall \lambda \in \mathbb{R} .
$$

The Airy function $\operatorname{Ai}(z), z \in \mathbb{C}$ is entire and satisfies (see (4.01)-(4.05) from [39]):

$$
\begin{gathered}
\operatorname{Ai}^{\prime \prime}(z)=z \operatorname{Ai}(z), \\
\operatorname{Ai}(-z)=e^{i \pi / 3} \operatorname{Ai}\left(z e^{i \pi / 3}\right)+e^{-i \pi / 3} \operatorname{Ai}\left(z e^{-i \pi / 3}\right),
\end{gathered}
$$

and it obeys the following asymptotics, as $|z| \rightarrow \infty$ uniformly in $\arg z$ for any fixed $\varepsilon>0$ :

$$
\begin{gathered}
\operatorname{Ai}(z)=\frac{1}{2} z^{-\frac{1}{4}} e^{-\frac{2}{3} z^{\frac{3}{2}}}\left(1+O\left(z^{-\frac{3}{2}}\right)\right), \quad \text { if } \quad|\arg z|<\pi-\varepsilon, \\
\operatorname{Ai}(-z)=z^{-\frac{1}{4}}\left[\sin \vartheta+O\left(z^{-\frac{3}{2}} e^{|\operatorname{Im} \vartheta|}\right)\right], \quad \text { if } \quad|\arg z| \leqslant \varepsilon, \quad \vartheta=\frac{2}{3} z^{\frac{3}{2}}+\frac{\pi}{4} .
\end{gathered}
$$


Introduce the space $L^{p}(\mathbb{R})$ equipped by the norm $\|f\|_{p}=\left(\int_{\mathbb{R}}|f(x)|^{p} d x\right)^{\frac{1}{p}} \geqslant 0$. For the scalar product $\langle f, f\rangle$ in $L^{2}(\mathbb{R})$ and we have $\langle f, f\rangle=\|f\|^{2}=\|f\|_{2}^{2}$. Define the linear functional $\Psi(\lambda): L^{2}(\mathbb{R}) \rightarrow \mathbb{C}$, by

$$
\Psi(\lambda) f=\left\langle f, G(\lambda)|V|^{\frac{1}{2}}\right\rangle=\frac{1}{\sqrt{\pi}} \int_{\mathbb{R}} \operatorname{Ai}(x-\lambda)|V(x)|^{\frac{1}{2}} f(x) d x \quad \forall \lambda \in \mathbb{R},
$$

which is bounded, since $\mathrm{Ai}(\cdot) \in L^{\infty}(\mathbb{R})$ by (2.21) and $|V|^{\frac{1}{2}} \in L^{2}(\mathbb{R})$ under Condition $\mathrm{V}$.

Lemma 2.1. Let the potential $V$ satisfy Condition $V$ and let $\tau \in[0,1], \lambda, \mu \in \mathbb{R},|\lambda-\mu| \leqslant 1$. Then

i) The linear functional $\Psi(\lambda): L^{2}(\mathbb{R}) \rightarrow \mathbb{C}$, defined by (2.22) is bounded and satisfies

$$
\begin{gathered}
\|\Psi(\lambda)\| \leqslant \frac{C}{(1+|\lambda|)^{\frac{1}{4}}}, \\
\|\Psi(\lambda)-\Psi(\mu)\| \leqslant \frac{C|\lambda-\mu|^{\tau}}{(1+|\lambda|)^{\frac{1}{4}-\frac{\tau}{2}}} .
\end{gathered}
$$

ii) Moreover, $\Omega(\lambda)=\Psi(\lambda)^{*} \Psi(\lambda), \lambda \in \mathbb{R}$ is a rank one operator on $L^{2}(\mathbb{R})$ satisfying

$$
\begin{gathered}
\|\Omega(\lambda)\|_{\mathcal{B}_{1}} \leqslant \frac{C}{(1+|\lambda|)^{\frac{1}{2}}}, \\
\|\Omega(\lambda)-\Omega(\mu)\|_{\mathcal{B}_{1}} \leqslant \frac{C|\lambda-\mu|^{\tau}}{(1+|\lambda|)^{\frac{1-\tau}{2}}} .
\end{gathered}
$$

Here the constant $C$ in (2.23)-(2.26) depends on $V$ only.

iii) Let $q, q_{1}$ be multiplication operators by functions $q, q_{1} \in L^{2}(\mathbb{R})$ respectively. Then for all $\operatorname{Im} z \neq 0$ the following holds true:

$$
q R_{0}(z), \quad q R(z) \in \mathcal{B}_{2}, \quad \text { and } \quad q R_{0}(z) q_{1}, \quad q R(z) q_{1} \in \mathcal{B}_{1}
$$

and in particular, $V R_{0}(z) \in \mathcal{B}_{2}$.

Proof. In order to prove the lemma we need a following simple estimate:

$$
\int_{\mathbb{R}} \frac{|V(x)| d x}{(1+|x-\lambda|)^{a}} \leqslant\|V\|_{2} \frac{\gamma(1+\gamma)^{a}}{\left(1+\frac{|\lambda|}{2}\right)^{a}}, \quad \forall(a, \lambda) \in\left[0, \frac{1}{2}\right] \times \mathbb{C} .
$$

We have

$$
\int_{\mathbb{R}} \frac{|V(x)| d x}{(1+|x-\lambda|)^{a}} \leqslant\|V\|_{2} J(\lambda), \quad J(\lambda)^{2}=\int_{0}^{\gamma} \frac{d x}{(1+|x-\lambda|)^{2 a}} .
$$

We will estimate $J(\lambda)$ : firstly, if $|\lambda| \leqslant 2 \gamma$, then $J^{2}(\lambda) \leqslant \gamma$; secondly, if $|\lambda| \geqslant 2 \gamma$, then

$$
J(\lambda)^{2} \leqslant \int_{0}^{\gamma} \frac{d x}{\left(1+\frac{|\lambda|}{2}\right)^{2 a}}=\frac{\gamma}{\left(1+\frac{|\lambda|}{2}\right)^{2 a}} \leqslant \frac{2 \gamma}{(1+|\lambda|)^{2 a}},
$$

which gives (2.28).

i) Let $f \in L^{2}(\mathbb{R})$. The asymptotics (2.21) and the estimate (2.28) imply

$$
\begin{array}{r}
|\Psi(\lambda) f|^{2}=\left.\left.\frac{1}{\pi}\left|\int_{\mathbb{R}} \operatorname{Ai}(x-\lambda)\right| V(x)\right|^{\frac{1}{2}} f(x) d x\right|^{2} \leqslant C\left(\int_{\mathbb{R}} \frac{|V(x)|^{\frac{1}{2}}|f(x)| d x}{(1+|x-\lambda|)^{\frac{1}{4}}}\right)^{2} \\
\leqslant C\|f\|^{2} \int_{\mathbb{R}} \frac{|V(x)| d x}{(1+|x-\lambda|)^{\frac{1}{2}}} \leqslant \frac{C_{1}\|f\|^{2}}{(1+|\lambda|)^{\frac{1}{2}}}
\end{array}
$$


for some constants $C, C_{1}$. This yields (2.23). Next, we show (2.24). Asymptotics (2.21) entails

$$
|\operatorname{Ai}(\lambda)-\operatorname{Ai}(\mu)| \leqslant \frac{C|\lambda-\mu|^{\tau}}{(1+|\lambda|)^{\frac{1}{4}-\frac{\tau}{2}}}, \quad \forall \lambda, \mu \in \mathbb{R},|\lambda-\mu| \leqslant 1 .
$$

Then similar arguments as above give (with $t=\frac{1}{4}-\frac{\tau}{2}$ )

$$
\begin{aligned}
|\Psi(\lambda) f-\Psi(\mu) f| & =\left.\frac{1}{\sqrt{\pi}}\left|\int_{\mathbb{R}}(\operatorname{Ai}(x-\lambda)-\operatorname{Ai}(x-\mu))\right| V(x)\right|^{\frac{1}{2}} f(x) d x \mid \\
& \leqslant C_{0}|\lambda-\mu|^{\tau} \int_{\mathbb{R}} \frac{|V(x)|^{\frac{1}{2}}|f(x)| d x}{(1+|x-\lambda|)^{\frac{1}{4}-\frac{\tau}{2}}} \leqslant \frac{C_{2}|\lambda-\mu|^{\tau}}{(1+|\lambda|)^{\frac{1}{4}-\frac{\tau}{2}}}\|f\|,
\end{aligned}
$$

for some constants $C, C_{1}$. This yields (2.24). The results of ii) follow from i).

iii) For $\operatorname{Im} z \neq 0$ and $q \in L^{2}(\mathbb{R})$ due to (2.21) we obtain

$$
\left\|\left|R_{0}(z)\right|^{\frac{1}{2}} q\right\|_{\mathcal{B}_{2}}^{2}=\int_{\mathbb{R}^{2}} \frac{\operatorname{Ai}^{2}(x-p)|q(x)|^{2}}{\pi|p-z|} d x d p<\int_{\mathbb{R}^{2}} \frac{C|q(x)|^{2} d x d p}{|p-z|(1+|p-x|)^{\frac{1}{2}}}<\infty,
$$

which yields $q R_{0}(z), V R_{0}(z) \in \mathcal{B}_{2}$ and the equality of the domains $\mathscr{D}\left(H_{0}\right)=\mathscr{D}(H)$. From (2.33) we deduce that

$$
q R_{0}(z) q_{1}=\left(q\left|R_{0}(z)\right|^{\frac{1}{2}}\right)\left(R_{0}(z)^{\frac{1}{2}} q_{1}\right) \in \mathcal{B}_{1}
$$

Combining these results with the identity $R=R_{0}-R_{0} V R$ we arrive at (2.27).

2.3. Estimates on $Y_{0}$. In order to estimate the operator-valued functions $Y(\lambda), Y_{0}(\lambda), \lambda \in \overline{\mathbb{C}}_{ \pm}$ in terms of the trace class norm $\mathcal{B}_{1}$ we need some additional definitions.

Let $\mathcal{H}$ be a Banach space equipped with the norm $\|\cdot\|_{\mathcal{H}}$. For any $\vartheta>0, \tau \in(0,1)$ we introduce the Banach space $\mathfrak{X}_{\vartheta, \tau}(\mathbb{R})=\mathfrak{X}_{\vartheta, \tau}(\mathbb{R}, \mathcal{H})$ of the functions $f: \mathbb{R} \rightarrow \mathcal{H}$ equipped with the norm:

$$
\|f\|_{\mathfrak{x}_{\vartheta, \tau}(\mathbb{R})}=\sup _{t \in \mathbb{R},|h| \leqslant 1}(1+|t|)^{\vartheta}\left(\|f(t)\|_{\mathcal{H}}+\frac{\|f(t+h)-f(t)\|_{\mathcal{H}}}{|h|^{\tau}}\right)<\infty
$$

and the Banach space $\mathfrak{X}_{\vartheta, \tau}\left(\mathbb{C}_{ \pm}\right)=\mathfrak{X}_{\vartheta, \tau}\left(\mathbb{C}_{ \pm}, \mathcal{H}\right)$ of the functions $F: \mathbb{C}_{ \pm} \rightarrow \mathcal{H}$ equipped with the norm:

$$
\|F\|_{\mathfrak{x}_{\vartheta, \tau}\left(\mathbb{C}_{ \pm}\right)}=\sup _{\lambda, \mu \in \mathbb{C}_{+},|\lambda-\mu|<1}(1+|\lambda|)^{\vartheta}\left(\|F(\lambda)\|_{\mathcal{H}}+\frac{\|F(\lambda)-F(\mu)\|_{\mathcal{H}}}{|\lambda-\mu|^{\tau}}\right)<\infty .
$$

We recall Privalov's Lemma. Privalov actually proved his lemma for a certain contour and for scalar functions. Faddeev (see Lemma 3.1 in [10]) proved a version for Hilbert space valued functions, where the contour is the real line, see also [2] about the Hilbert transformation.

Lemma 2.2. (Privalov) Assume that $f: \mathbb{R} \rightarrow \mathcal{H}$ belongs to the Banach space $\mathfrak{X}_{\vartheta, \tau}(\mathbb{R}, \mathcal{H})$ for some $\vartheta>0, \tau \in(0,1)$ and for some Banach space $\mathcal{H}$. Then the function $F$ given by

$$
F(z)=\int_{\mathbb{R}} \frac{f(t)}{t-z} d t, \quad z \in \mathbb{C}_{+},
$$

is analytic in $\mathbb{C}_{+}$and continuous up to the real line. Moreover, it is bounded as a map $f \rightarrow F$ from $\mathfrak{X}_{\vartheta, \tau}(\mathbb{R}, \mathcal{H})$ into $\mathfrak{X}_{\vartheta_{1}, \tau}\left(\mathbb{C}_{ \pm}, \mathcal{H}\right)$, for any $\vartheta_{1}<\vartheta$, and satisfies

$$
\|F\|_{\mathfrak{X}_{\vartheta_{1}, \tau}\left(\mathbb{C}_{ \pm}, \mathcal{H}\right)} \leqslant C\|f\|_{\mathfrak{X}_{\vartheta, \tau}(\mathbb{R}, \mathcal{H})}, \quad \forall \quad \vartheta_{1}<\vartheta,
$$


where the constant $C=C\left(\vartheta_{1}, \vartheta, \tau\right)$ depends on $\vartheta_{1}, \vartheta$ and $\tau$ only.

We apply Privalov's Lemma 2.2 to study the sandwiched resolvents $Y_{0}(\cdot)$ and $Y(\cdot)$.

Lemma 2.3. Let the potential $V$ satisfy Condition $\mathrm{V}$ and let $\vartheta<\frac{1-\tau}{2}, 0<\tau<1$. Then i) The operator-valued functions $I+Y_{0}$ and $\left(I+Y_{0}\right)^{-1}$ are uniformly Hölder on $\overline{\mathbb{C}}_{+}$and

$$
\left(I+Y_{0}(\lambda)\right)^{-1} \in \mathcal{B}, \quad \forall \lambda \in \overline{\mathbb{C}}_{ \pm},
$$

Moreover, let $F=Y_{0}$ or $F=Y$. Then the operator-valued function $F$ is analytic on $\mathbb{C}_{ \pm}$and continuous up to the real line in the $\mathcal{B}_{1}$-norm and satisfies

$$
\|F\|_{\mathfrak{X}_{\vartheta, \tau}\left(\mathbb{C}_{ \pm}, \mathcal{B}_{1}\right)}<\infty \text {. }
$$

ii) Moreover, the function $D_{ \pm}$is analytic in $\mathbb{C}_{ \pm}$and satisfies

$$
D_{ \pm}-1 \in \mathfrak{X}_{\vartheta, \tau}\left(\mathbb{C}_{ \pm}\right) \text {. }
$$

Proof. i) Introduce the Hilbert space $\mathfrak{S}_{a}, a \geqslant 0$ of Hilbert-Schmidt operators $X$ equipped with the norm

$$
\|X\|_{\mathfrak{S}_{a}}^{2}=\sum_{n \geqslant 1} n^{2 a} \sigma_{n}^{2}
$$

where the non-negative numbers $\sigma_{1} \geqslant \sigma_{2} \geqslant \sigma_{3} \ldots$ are eigenvalues of the operator $\left(X^{*} X\right)^{\frac{1}{2}} \geqslant 0$. Note that $\|X\|_{\mathcal{B}_{1}} \leqslant C_{a}\|X\|_{\mathfrak{S}_{a}}$ for all $a>\frac{1}{2}$, where the constant is given by $C_{a}^{2}=\sum_{n \geqslant 1} n^{-2 a}$.

In the free case the operator $Y_{0}(\lambda)$ has the form

$$
\begin{array}{r}
Y_{0}(\lambda)=\int_{\mathbb{R}} \frac{\Omega(t)}{t-\lambda} d t, \quad \lambda \in \mathbb{C}_{+}, \\
\Omega(t)=\Psi(t)^{*} \Psi(t) V_{S}, \quad V_{S}=\operatorname{sign} V .
\end{array}
$$

Here, due to Lemma [2.1, the function $\Omega(t), t \in \mathbb{R}$ is a rank one operator-valued function and, in particular, $\Omega(t) \in \mathfrak{S}_{1}, t \in \mathbb{R}$. Thus Lemma 2.1 shows that $\Omega \in \mathfrak{X}_{\vartheta, \tau}\left(\mathbb{R}, \mathfrak{S}_{1}\right)$ for any $\vartheta=\frac{1-\tau}{2}, \quad 0<\tau<1$. Then Privalov's Lemma 2.2 yields (2.39) for $Y_{0}$.

The operator $I+Y_{0}(\lambda)$ is invertible for all $\lambda \in \mathbb{C}_{ \pm}$, since $H_{0}$ is self-adjoint and satisfies (2.27). Moreover, it is standard fact that the operator $I+Y_{0}(\lambda \pm i 0)$ is invertible for all $\lambda \in \mathbb{R}$. In fact, this follows from (2.23), (2.24). These remarks and the properties of $Y_{0}$ imply that the operator-valued functions $I+Y_{0}$ and $\left(I+Y_{0}\right)^{-1}$ are analytic in $\mathbb{C}_{+}$, continuous up to the real line and uniformly Hölder continuous in $\overline{\mathbb{C}}_{+}$.

Consider the case $F=Y$. Using the identity $\left(I+Y_{0}\right) Y=Y_{0}$ and the properties of $Y_{0}$ and $I+Y_{0}$ described above we obtain the proof of i).

ii) Due to (2.39) the function $D_{ \pm}(\lambda)=\operatorname{det}\left(I+Y_{0}(\lambda)\right)$ is well-defined and analytic in $\mathbb{C}_{ \pm}$. Moreover, using (2.6) we have

$$
\left|\operatorname{det}\left(I+Y_{0}(\lambda)\right)-1\right| \leqslant\left\|Y_{0}(\lambda)\right\|_{\mathcal{B}_{1}} e^{1+\left\|Y_{0}(\lambda)\right\|_{\mathcal{B}_{1}}}
$$

and adding (2.39), we obtain (2.40).

Lemma 2.4. Let $V$ satisfy Condition $\mathrm{V}$ and let $\tau<\frac{1}{2}$. Then

$$
\left|\operatorname{Tr} Y_{0}^{n}(\lambda)\right| \leqslant\left\|Y_{0}^{n}(\lambda)\right\|_{\mathcal{B}_{1}} \leqslant \frac{C}{|\lambda|^{n \tau}} \quad \forall n \geqslant 1,
$$


where $\lambda \in \overline{\mathbb{C}}_{+},|\lambda|>1$ and $C=C(\tau, V)$ depending on $\tau, V$. Let, in addition, $V$ satisfy Condition C. Then

$$
\operatorname{Tr} Y_{0}(\lambda)=\frac{i}{2 \sqrt{\lambda}} V_{0}+\frac{O(1)}{\lambda}
$$

as $|\lambda| \rightarrow \infty, \lambda \in \overline{\mathbb{C}}_{+}$, where $V_{0}=\int_{\mathbb{R}} V(x) d x$ uniformly in $\arg \lambda \in[0, \pi]$.

Proof. For $\lambda \in \overline{\mathbb{C}}_{+}$, due to (2.39) we have

$$
\left|\operatorname{Tr} Y_{0}^{n}(\lambda)\right| \leqslant\left\|Y_{0}^{n}(\lambda)\right\|_{\mathcal{B}_{1}} \leqslant\left\|Y_{0}(\lambda)\right\|_{\mathcal{B}_{1}}^{n} \leqslant \frac{C_{\tau}}{|\lambda|^{n \tau}}
$$

where the constant $C=C(\varepsilon, V)$ does not depend on $\lambda$. We show (2.43) in Lemma 6.1,

\section{Determinants And S-Matrix}

3.1. The Determinants. We discuss the determinant $D_{ \pm}(\lambda), \lambda \in \overline{\mathbb{C}}_{ \pm}$, when the potential $V$ satisfies Condition V. In this case we have (2.27) and this gives the identity

$$
\frac{D_{ \pm}^{\prime}(\lambda)}{D_{ \pm}(\lambda)}=\operatorname{Tr} R_{0}(\lambda) V R(\lambda)=\operatorname{Tr}\left(R_{0}(\lambda)-R(\lambda)\right), \quad \lambda \in \mathbb{C}_{ \pm}
$$

which is well-known for large class of operators. Due to (2.39) the operator-valued function $Y_{0}(\lambda)$ attains value in $\mathcal{B}_{1}$ and belongs to the class $\mathfrak{X}_{\vartheta, \tau}\left(\mathbb{C}_{ \pm}, \mathcal{B}_{1}\right)$ for any $\vartheta<\frac{1-\tau}{2}$ and $0<\tau<1$. Recall that we define $\log D_{ \pm}(\lambda)$, by $\log D_{ \pm}(\lambda)=o(1)$ as $|\lambda| \rightarrow \infty, \lambda \in \mathbb{C}_{ \pm}$, since $D_{ \pm}(\lambda) \neq 0$ for all $\lambda \in \overline{\mathbb{C}}_{ \pm}$and $\left\|Y_{0}(\lambda)\right\|_{\mathcal{B}_{1}}=o(1)$ as $|\lambda| \rightarrow \infty$ and there exists $r_{0}>0$ such that

$$
\sup _{\lambda \in \mathbb{C}_{+},|\lambda| \geqslant r_{0}}\left\|Y_{0}(\lambda)\right\|_{\mathcal{B}_{1}}<\frac{1}{2} .
$$

Then using (2.3) $-(2.6)$, (2.39) we obtain

$$
\log D_{ \pm} \in \mathfrak{X}_{\vartheta, \tau}\left(\mathbb{C}_{ \pm}\right), \quad \forall \vartheta<\frac{1-\tau}{2}, 0<\tau<1
$$

It is well-known (see [41]) that, under the condition (3.2), the function $\log D_{ \pm}(\lambda)$ satisfies

$$
-\log D_{ \pm}(\lambda)=\sum_{n=1}^{\infty} \frac{1}{n} \operatorname{Tr}\left(-Y_{0}(\lambda)\right)^{n}
$$

for any $\lambda \in \overline{\mathbb{C}}_{ \pm},|\lambda|>r_{0}$, where the series converges absolutely and uniformly. Then using (3.4) and (3.2) for some $r_{0}>0$ and any $\tau<\frac{1}{2}$ we obtain

$$
\left|\log D_{ \pm}(\lambda)+\sum_{n=1}^{N} \frac{1}{n} \operatorname{Tr}\left(-Y_{0}(\lambda)\right)^{n}\right| \leqslant \frac{\left\|Y_{0}(\lambda)\right\|_{\mathcal{B}_{1}}^{N+1}}{N+1} \leqslant \frac{C}{|\lambda|^{(N+1) \tau}}, \quad \forall N \geqslant 0
$$


3.2. The scattering matrix. Recall that the S-matrix $S(\lambda)$ is a scalar function of $\lambda \in \mathbb{R}$, acting as multiplication in the fiber spaces $\mathbb{C}=\mathscr{H}_{\lambda}$. Thus $|S(\lambda)|=1$ for all $\lambda \in \mathbb{R}$ we have

$$
S(\lambda)=e^{-2 \pi i \phi_{s c}(\lambda)}, \quad \lambda \in \mathbb{R} .
$$

The stationary representation for the scattering matrix has the form (see e.g. [48]):

$$
\begin{aligned}
& S(\lambda)=I-2 \pi i \mathcal{A}(\lambda), \quad \lambda \in \mathbb{R} ; \quad \mathcal{A}=\mathcal{A}_{0}-\mathcal{A}_{1}, \\
& \mathcal{A}_{0}(\lambda)=\Psi(\lambda) V_{S} \Psi^{*}(\lambda), \quad \mathcal{A}_{1}(\lambda)=\Psi(\lambda) V_{S} Y(\lambda+i 0) \Psi^{*}(\lambda), \\
& \Psi(\lambda)=G(\lambda)|V|^{\frac{1}{2}}, \quad V_{S}=\operatorname{sign} V,
\end{aligned}
$$

where $G$ is given by (2.18). Note that due to (2.39) the operator $Y(\lambda \pm i 0)$ is continuous in $\lambda \in \mathbb{R}$. We shall represent $S(\lambda)$ in terms of $D_{ \pm}(\lambda)$.

Lemma 3.1. Let $V$ satisfy Condition $\mathrm{V}$. Then the scattering amplitude $\mathcal{A}(\lambda)$ is a continuous scalar function of $\lambda \in \mathbb{R}$ and satisfies

$$
\begin{gathered}
\mathcal{A}_{0}(\lambda)=\int_{\mathbb{R}} \operatorname{Ai}(x-\lambda)^{2} V(x) d x \quad \forall \lambda \in \mathbb{R}, \\
\mathcal{A}_{0}(\cdot) \in \mathfrak{X}_{\frac{1-\tau}{2}, \tau}(\mathbb{R}, \mathbb{C}), \\
\mathcal{A}_{1}(\cdot) \in \mathfrak{X}_{\varepsilon, \tau}(\mathbb{R}, \mathbb{C}) \quad \forall \varepsilon<1-\tau,
\end{gathered}
$$

for any $\tau \in(0,1)$. Moreover, the functions $S(\lambda), \phi_{s c}(\lambda)$ are continuous in $\lambda \in \mathbb{R}$ and satisfy asymptotics

$$
\begin{gathered}
S(\lambda)-1=O\left(\lambda^{-\frac{1}{2}}\right), \quad \phi_{s c}(\lambda)=O\left(\lambda^{-\frac{1}{2}}\right) \quad \text { as } \quad \lambda \rightarrow \pm \infty, \\
\phi_{s c}(\lambda)=\frac{1}{\pi} \arg D_{+}(\lambda), \quad \lambda \in \overline{\mathbb{C}}_{+},
\end{gathered}
$$

and the identities (1.6) which uniquely defines $\phi_{s c}$ by (3.6), continuity and the asymptotics (3.11).

Proof. The definitions of $\mathcal{A}_{0}$ and $\Psi$ (see (3.7)) give (3.8). Relation (3.9) follows from Lemma 2.1. Relation (3.10) follows from Lemma 2.1 and (2.39), since $Y(\cdot) \in \mathfrak{X}_{\vartheta, \tau}\left(\mathbb{C}_{ \pm}, \mathcal{B}_{1}\right)$, for any $\vartheta<\frac{1-\tau}{2}, \tau \in(0,1)$ due to (2.39).

Next, we show (1.6) . Recall that $S(\lambda)$ satisfies (3.7) and that we have the standard identity

$$
Y_{0}(\lambda+i 0)-Y_{0}(\lambda-i 0)=2 \pi i \Psi(\lambda)^{*} \Psi(\lambda) V_{S}, \quad \lambda \in \mathbb{R} .
$$

Then (3.7), (2.2) give

$$
\begin{aligned}
& \operatorname{det} S(\lambda)=\operatorname{det}\left(I-2 \pi i \Psi(\lambda) V_{S} J(\lambda+i 0) \Psi(\lambda)^{*}\right) \\
& =\operatorname{det}\left(I-2 \pi i \Psi(\lambda)^{*} \Psi(\lambda) V_{S} J(\lambda+i 0)\right)=\operatorname{det} J(\lambda+i 0) \operatorname{det}\left(J_{0}(\lambda+i 0)-2 \pi i \Psi(\lambda)^{*} \Psi(\lambda) V_{S}\right) \\
& =\operatorname{det} J(\lambda+i 0) \operatorname{det}\left(J_{0}(\lambda+i 0)-Y_{0}(\lambda+i 0)+Y_{0}(\lambda-i 0)\right) \\
& =\operatorname{det} J(\lambda+i 0) \operatorname{det} J_{0}(\lambda-i 0),
\end{aligned}
$$

which together with (2.12) yields (1.6) since

$$
\operatorname{det} S(\lambda)=\operatorname{det} J(\lambda+i 0) \operatorname{det} J_{0}(\lambda-i 0)=\frac{\operatorname{det} J_{0}(\lambda-i 0)}{\operatorname{det} J_{0}(\lambda+i 0)}=\frac{D_{-}(\lambda-i 0)}{D_{+}(\lambda+i 0)}=\frac{\bar{D}_{+}(\lambda+i 0)}{D_{+}(\lambda+i 0)} \text {. }
$$


This yields (3.12) and adding the relation (3.3) we obtain $\phi_{s c}(\lambda)=O\left(|\lambda|^{-a}\right)$ as $\lambda \rightarrow \pm \infty$ for any $a<\frac{1}{2}$. Substituting estimates (2.39) and (2.23) into (3.7) we obtain $S(\lambda)-1=O\left(\lambda^{-\frac{1}{2}}\right)$ as $\lambda \rightarrow \pm \infty$. As both $\phi_{s c}(\lambda)$ and $S(\lambda)$ are continuous in $\lambda$, formula (3.6) determines $\phi_{s c}(\lambda)$ by $\phi_{s c}(\lambda)=\frac{i}{2 \pi} \log S(\lambda)$ and the asymptotics $\phi_{s c}(\lambda)=O\left(\lambda^{-\frac{1}{2}}\right)$ as $\lambda \rightarrow \pm \infty$. All together this proves Lemma 3.1 .

Proof of Theorem 1.1. Due to Lemma 2.3 the function $D_{ \pm}$is analytic in $\mathbb{C}_{ \pm}$, continuous up to the real line. Asymptotics (3.5) and (2.43) yield (1.8), which gives (1.9).

Proposition 3.2. Let $V$ satisfy Condition C. Then the following trace formulas hold true:

$$
\begin{gathered}
\int_{\mathbb{R}} V(x) d x=\frac{2}{\pi} \int_{\mathbb{R}} \operatorname{Re} \frac{\log D_{+}(\lambda+i 0)}{\sqrt{\lambda+i 0}} d \lambda, \\
\lim _{r \rightarrow \infty} \int_{-r}^{r} \operatorname{Im} \frac{\log D_{+}(\lambda+i 0)}{\sqrt{\lambda+i 0}} d \lambda=0 .
\end{gathered}
$$

Proof. Define a contour : $\Gamma_{r}=c_{r} \cup(-r, r)$, where $c_{r}=\{|\lambda|=r\} \cap \mathbb{C}_{+}$for large $r \rightarrow+\infty$. The function $f(\lambda)=i \frac{\log D_{+}(\lambda)}{\sqrt{\lambda}}$ is analytic in the upper-half-plane and continuous up to the real line without zero. This gives

$$
0=\int_{\Gamma_{r}} f(\lambda) d \lambda=I_{r}+I_{r}^{+}, \quad I_{r}^{+}=\int_{c_{r}} f(\lambda) d \lambda, \quad I_{r}=\int_{-r}^{r} f(\lambda) d \lambda
$$

Due to asymptotics (1.8) $f(\lambda)=\frac{i V_{0}+O\left(\lambda^{-\varepsilon}\right)}{2 \lambda}$ as $|\lambda| \rightarrow \infty$ uniformly in $\arg \lambda \in[0, \pi]$ for some $\varepsilon>0$, we obtain

$$
I_{r}^{+}=\int_{c_{r}} f(\lambda) d \lambda=\frac{i V_{0}}{2} \int_{c_{r}} \frac{d \lambda}{\lambda}+o(1)=-\frac{\pi}{2} V_{0}+o(1)
$$

and

$$
I_{r}=\int_{-r}^{r} f(\lambda) d \lambda=\frac{\pi}{2} V_{0}+o(1)
$$

as $r \rightarrow \infty$, and here

$$
\operatorname{Re} f(\lambda+i 0) \in L^{1}(\mathbb{R}), \quad \operatorname{Im} f(\lambda+i 0)=\frac{V_{0}}{2 \lambda}+O\left(\lambda^{-\varepsilon-1}\right) \quad \text { as } \pm \lambda \rightarrow \infty .
$$

Combining all relations (3.16)-(3.18) we obtain (3.14)-(3.15).

Remark. We recall that trace formulas are important to study non linear equations, inverse problems, spectral theory, etc. see [9], [11, [25], [34] and references therein. The complete asymptotic expansion of the scattering phase (the spectral shift function) $\phi_{s c}$ at high energies and a sequence of trace formulas for 3-dim perturbed Stark operators were determined by Korotyaev-Pushnitski 31].

\section{Analyticity of $\mathcal{A}_{0}$}

4.1. Estimates on Airy functions. In this section we assume that the potential $V$ satisfies Condition V. Recall that by (3.7), the functional $\Psi(\lambda): L^{2}(\mathbb{R}) \rightarrow \mathbb{C}$ and its adjoint $\Psi^{*}(\lambda)$ : $\mathbb{C} \rightarrow L^{2}(\mathbb{R})$ for $\lambda \in \mathbb{R}$ are given by

$$
\Psi(\lambda) f=\int_{\mathbb{R}} \operatorname{Ai}(x-\lambda)|V(x)|^{\frac{1}{2}} f(x) d x, \quad \Psi^{*}(\lambda) c=\operatorname{Ai}(x-\lambda)|V(x)|^{\frac{1}{2}} c, \quad \lambda \in \mathbb{R},
$$


where $(f, c) \in L^{2}(\mathbb{R}) \times \mathbb{C}$. Assuming that $V$ has compact support, these mappings are bounded on the real line and have analytic extensions from $\mathbb{R}$ onto the whole complex plane. We remark that supp $V$ being bounded from below suffices to render $\Psi(\lambda)$ analytic. To prove our estimates supp $V$ being compact is, however, helpful. Thus $\Psi^{*}(\bar{\lambda})$ can be identified with the function $\mathrm{Ai}(\cdot-\lambda)|V(\cdot)|^{\frac{1}{2}}$ in $L^{2}(\mathbb{R})$, which is analytic in $\lambda \in \mathbb{C}$.

In order to estimate $\Psi, \Psi^{*}$, we need the asymptotics of the Airy function from (2.21). Furthermore we have

$$
\left\{\begin{array}{l}
(x-\lambda)^{-\frac{1}{4}}=(-\lambda)^{-\frac{1}{4}}\left(1+O\left(|\lambda|^{-1}\right),\right. \\
(x-\lambda)^{\frac{3}{2}}=(-\lambda)^{\frac{3}{2}}+\frac{3}{2} x(-\lambda)^{\frac{1}{2}}+O\left(|\lambda|^{-\frac{1}{2}}\right)
\end{array} \quad, \quad|\arg \lambda| \geqslant \varepsilon,\right.
$$

and

$$
\left\{\begin{array}{l}
(\lambda-x)^{-\frac{1}{4}}=\lambda^{-\frac{1}{4}}\left(1+O\left(|\lambda|^{-1}\right),\right. \\
(\lambda-x)^{\frac{3}{2}}=\lambda^{\frac{3}{2}}-\frac{3}{2} x \lambda^{\frac{1}{2}}+O\left(|\lambda|^{-\frac{1}{2}}\right)
\end{array}, \quad|\arg \lambda| \leqslant \varepsilon,\right.
$$

locally uniformly in $x \in \mathbb{R}$, as $|\lambda| \rightarrow \infty$. We will use these estimates in order to determine asymptotics of Airy functions. Asymptotics (4.2), (2.21) with $z=x-\lambda$ and straightforward calculation give the following symptotics (4.4), (4.5):

i) Let $|\arg \lambda| \geqslant \varepsilon$ and let $\zeta=\sqrt{-\lambda},|\arg \zeta| \leqslant \frac{\pi-\varepsilon}{2}$. Then as $|\lambda| \rightarrow \infty$ one has

$$
\operatorname{Ai}(x-\lambda)^{2}=\frac{1}{4 \zeta} e^{-\left(\frac{4}{3} \zeta^{3}+2 x \zeta\right)}\left(1+\frac{O(1)}{\zeta}\right)
$$

and, in particular,

$$
|\operatorname{Ai}(x-\lambda)|^{2}=\frac{1}{4|\lambda|^{\frac{1}{2}}} e^{-\operatorname{Re}\left(\frac{4}{3} \zeta^{3}+2 x \zeta\right)}\left(1+\frac{O(1)}{|\lambda|^{1 / 2}}\right) .
$$

Moreover, for the case $|\arg \lambda| \leqslant \varepsilon$ using (4.3), we set $X=\frac{4}{3}(\lambda-x)^{\frac{3}{2}}$ and obtain after short calculation

$$
X=\frac{4}{3} \lambda^{\frac{3}{2}}-2 x \lambda^{\frac{1}{2}}+O\left(|\lambda|^{-\frac{1}{2}}\right)=\eta+O\left(|\lambda|^{-\frac{1}{2}}\right) \quad \text { as } \quad|\lambda| \rightarrow \infty, \quad|\arg \lambda| \leqslant \varepsilon .
$$

where $\eta=\frac{4}{3} \zeta^{3}-2 x \zeta$. Substituting this into (2.21) for $|\arg \lambda| \leqslant \varepsilon$, we obtain the following asymptotics and the estimate:

ii) Let $|\arg \lambda| \leqslant \varepsilon, \zeta=\sqrt{\lambda},|\arg \zeta| \leqslant \frac{\varepsilon}{2}$ and let $|\lambda|$ be sufficiently large. Then

$$
\operatorname{Ai}(x-\lambda)^{2}=\frac{1+\sin \eta}{2 \zeta}+\frac{O\left(e^{|\operatorname{Im} \eta|}\right)}{\lambda},
$$

where $\eta=\frac{4}{3} \zeta^{3}-2 x \zeta$, and in particular, the following slightly weaker estimate holds true:

$$
|\operatorname{Ai}(x-\lambda)|^{2} \leqslant \frac{1}{|\lambda|^{\frac{1}{2}}} e^{|\operatorname{Im} \eta|}\left(1+\frac{O(1)}{|\lambda|^{\frac{1}{2}}}\right) .
$$

Note that all estimates 4.4)-4.8) are locally uniform in $x$ on bounded intervals. 
4.2. Estimates on the Born term $\mathcal{A}_{0}$. Now we are ready to study the Born term $\mathcal{A}_{0}$.

Lemma 4.1. Let $V$ satisfy Condition $V$ and let $\varepsilon>0$. Then the Born term $\mathcal{A}_{0}(\lambda), \lambda \in \mathbb{R}$ given by (3.8) has an analytic extension from the real line into the whole complex plane and satisfies as $|\lambda| \rightarrow \infty$ :

i) Let $|\arg \lambda| \geqslant \varepsilon$ and let $-i \zeta=\sqrt{-\lambda}, \quad\left|\arg \zeta-\frac{\pi}{2}\right| \leqslant \varepsilon$. Then

$$
\mathcal{A}_{0}(\lambda)=\frac{i}{4 \zeta} e^{-i \frac{4}{3} \zeta^{3}} \int_{0}^{\gamma} e^{i 2 x \zeta} V(x)\left(1+\frac{O(1)}{\zeta}\right) d x
$$

and

$$
\left|\mathcal{A}_{0}(\lambda)\right| \leqslant \frac{C}{|\zeta|} e^{\frac{4}{3} \operatorname{Re} \zeta^{3}}\|V\|_{L^{1}(0, \gamma)}
$$

for some absolute constants $C$.

ii) Let $|\arg \lambda| \leqslant \varepsilon$ and let $\zeta=\sqrt{\lambda},|\arg \zeta| \leqslant \varepsilon$. Then

$$
\mathcal{A}_{0}(\lambda)=\frac{1}{2 \zeta} \int_{0}^{\gamma} V(x)\left[1+\sin \eta+\frac{O\left(e^{|\operatorname{Im} \eta|}\right)}{\zeta}\right] d x
$$

where $\eta=\frac{4}{3} \zeta^{3}-2 x \zeta$ and

$$
\left|\mathcal{A}_{0}(\lambda)\right| \leqslant \frac{1}{2|\zeta|} \int_{0}^{\infty}|V(x)|\left[1+|\sin \eta|+\frac{O\left(e^{|\operatorname{Im} \eta|}\right)}{|\zeta|}\right] d x
$$

Let, in addition, $|\zeta| \geqslant 1+\gamma$ and $|\arg \zeta| \leqslant \frac{\pi}{6}$. Then

$$
\left|\mathcal{A}_{0}(\lambda)\right| \leqslant \frac{e^{\frac{4}{3}\left|\operatorname{Im} \zeta^{3}\right|}}{2|\zeta|} \int_{0}^{\gamma}|V(x)|\left(1+\frac{O(1)}{|\zeta|}\right) d x .
$$

Proof. i) Substituting (4.2), (4.4) in (3.8) we obtain (4.9) and (4.10).

ii) Using (4.5) we obtain (4.11), which yields (4.12). Let $\zeta=r e^{i \phi}$, where $|\zeta| \geqslant 1+\gamma$ and $|\phi| \leqslant \frac{\pi}{6}$. Then we have

$$
|\operatorname{Im} \eta|=\left|\operatorname{Im}\left(\frac{2}{3} \zeta^{3}-x \zeta\right)\right|=r\left|\frac{2}{3} r^{2} \sin 3 \phi-x \sin \phi\right| \leqslant \frac{2}{3} r^{3} \sin 3|\phi| .
$$

Substituting the last estimate into (4.11) we obtain (4.13).

\section{RESOnANCES AND S-MATRIX}

5.1. Analyticity of S-matrix. We discuss a meromorphic continuation of the S-matrix $S(\lambda)$ from the real line onto the whole complex plane.

Lemma 5.1. Let $V$ satisfy Condition $\mathrm{V}$ and let $\varepsilon>0$. Then

i) The functionals $\Psi(\lambda): L^{2}(\mathbb{R}) \rightarrow \mathbb{C}$ given by (4.1), and the mapping $\Psi^{*}(\bar{\lambda})=\Psi^{*}(\lambda)$, for all $\lambda \in \mathbb{R}$ have analytic extensions from the real line into the whole complex plane and satisfy

$$
\begin{gathered}
\|\Psi(\lambda)\|^{2}=\left\|\Psi^{*}(\bar{\lambda})\right\|^{2}=\int_{0}^{\gamma}|\operatorname{Ai}(x-\lambda)|^{2}|V(x)| d x, \\
\|\Psi(\lambda)\|^{2} \leqslant \frac{C}{(1+|\lambda|)^{\frac{1}{2}}} e^{\frac{4}{3}|\lambda|^{\frac{3}{2}}}
\end{gathered}
$$

for all $\lambda \in \mathbb{C}$ and for some constant $C=C(V)$. 
ii) The scattering amplitude $\mathcal{A}(\lambda)=\mathcal{A}_{0}(\lambda)-\mathcal{A}_{1}(\lambda)$, defined in (3.7) for $\lambda \in \mathbb{R}$, has an analytic extension from the real line into the whole upper half-plane satisfying

$$
\left|\mathcal{A}_{1}(\lambda)\right| \leqslant \frac{C_{a}}{(1+|\lambda|)^{a}}\|\Psi(\lambda)\|^{2} \quad \forall \quad \lambda \in \overline{\mathbb{C}}_{+},
$$

for any $a \in\left(0, \frac{1}{2}\right)$ and some constant $C=C_{a}$ depending on a.

Proof. i) Since the Airy function is entire, the functional $\Psi(\lambda): L^{2}(\mathbb{R}) \rightarrow \mathbb{C}$ given by (4.1), and the mapping $\Psi(\lambda)^{*}$, for all $\lambda \in \mathbb{R}$ have analytic extensions from the real line into the whole complex plane. The identities in (5.1) are obvious from the definitions of $\Psi, \Psi^{*}$. The proof of (5.2) is a repeatition of the proof of (4.13) and (4.9). In fact, substituting (4.5), (4.8) into (5.1) we obtain (5.2).

ii) The operators $\Psi, \Psi_{1}$ have analytic extensions from the real line into the whole complex plane and operator-valued function $Y(\lambda+i 0)$ also has an analytic extension from the real line into the upper-half plane. Then $\left.\mathcal{A}_{1}(\lambda)=\Psi(\lambda) V_{S} Y(\lambda+i 0)\right) \Psi^{*}(\lambda)$ has an analytic extension from the real line into the upper-half plane and thus $\mathcal{A}$ has so. Moreover, (2.39) gives

$$
\left|\mathcal{A}_{1}(\lambda)\right| \leqslant\|\Psi(\lambda)\|^{2}\|Y(\lambda)\| \leqslant C(1+|\lambda|)^{-a}\|\Psi(\lambda)\|^{2} \quad \forall \lambda \in \overline{\mathbb{C}}_{+},
$$

where $C=C(V)$ is some constant.

Lemma 5.2. Let $V$ satisfy Condition $\mathrm{C}$ with $V(0) \neq 0$ and let $\lambda=t^{2} e^{i \frac{\pi}{3}}, \quad \zeta=e^{i \frac{\pi}{6}} t$ as $t \rightarrow+\infty$. Then

$$
\begin{gathered}
\mathcal{A}_{0}(\lambda)=\frac{e^{i \frac{\pi}{3}+\frac{4}{3} t^{3}}}{4 t} \int_{0}^{\gamma} e^{(-1+i \sqrt{3}) t x} V(x)\left(1+\frac{O(1)}{t}\right) d x=\frac{e^{i \frac{2 \pi}{3}+\frac{4}{3} t^{3}}}{8 t^{2}}(V(0)+o(1)), \\
\|\Psi(\lambda)\|^{2} \leqslant e^{\frac{4}{3} t^{3}} \frac{C}{t^{2}}\|V\|, \\
\mathcal{A}(\lambda)=\frac{e^{i \frac{2 \pi}{3}+\frac{4}{3} t^{3}}}{8 t^{2}}(V(0)+o(1)) .
\end{gathered}
$$

Proof. Let $\sigma=1-i \sqrt{3}=2 e^{-i \frac{\pi}{3}}$ and let $\zeta=e^{i \frac{\pi}{6}} t$ as $t \rightarrow+\infty$. From (4.9) we have

$$
\begin{aligned}
\mathcal{A}_{0}(\lambda)=\frac{i e^{-i \frac{\pi}{6}}}{4 t} e^{\frac{4}{3} t^{3}} \mathcal{I}(\lambda), \quad \mathcal{I}(\lambda)=\int_{0}^{\gamma} e^{-\sigma t x} V(x)\left(1+\frac{O(1)}{t}\right) d x=\mathcal{I}_{1}(\lambda)+\mathcal{I}_{2}(\lambda), \\
\mathcal{I}_{1}(\lambda)=\int_{0}^{\gamma_{1}} e^{-\sigma t x} V(x)\left(1+\frac{O(1)}{t}\right) d x \\
\mathcal{I}_{2}(\lambda)=\int_{\gamma_{1}}^{\gamma} e^{-\sigma t x} V(x)\left(1+\frac{O(1)}{t}\right) d x=e^{-t \gamma_{1}}\|V\|_{1} O(1) .
\end{aligned}
$$

An integration by parts yields

$$
\begin{aligned}
& \int_{0}^{\gamma} e^{-\sigma t x} V(x) d x=-\left.\frac{1}{\sigma t} e^{-\sigma t x} V(x)\right|_{0} ^{\gamma}+\frac{1}{\sigma t} \int_{0}^{\gamma} e^{-\sigma t x} V^{\prime}(x) d x \\
= & \frac{1}{\sigma t}\left(V(0)-e^{-\sigma t \gamma} V(\gamma)+\int_{0}^{\gamma} e^{-\sigma t x} V^{\prime}(x) d x\right)=\frac{V(0)-o(1)}{\sigma t},
\end{aligned}
$$


since since $V$ is absolutely continuous, its derivative is in $L^{1}(\mathbb{R})$ and for $u \in L^{1}(0, \infty)$ the following asymptotics hold true

$$
\int_{0}^{\gamma} e^{-t x} u(x) d x=o(1) \quad \text { as } \quad t \rightarrow \infty .
$$

Moreover, (5.9) gives $\int_{0}^{\gamma} e^{-\sigma t x} V(x) d x=o(1)$. Combining all these estimates we obtain (5.5).

We consider $\|\Psi(\lambda)\|^{2}$ and we show (5.6). Let $\zeta=t e^{i \frac{\pi}{6}}$ as $t \rightarrow+\infty$. From (4.9) we have

$$
\|\Psi(\lambda)\|^{2}=\int_{0}^{\gamma}|\operatorname{Ai}(x-\lambda)|^{2}|V(x)| d x=\frac{1}{4 t} e^{\frac{4}{3} t^{3}} \int_{0}^{\gamma} e^{-t x}|V(x)|(1+o(1)) d x,
$$

and

$$
\begin{aligned}
\int_{0}^{\gamma} e^{-t x}|V(x)| d x & =\int_{0}^{\gamma_{1}} e^{-t x}|V(x)| d x+\int_{\gamma_{1}}^{\gamma} e^{-t x}|V(x)| d x \\
& \leqslant\|V\|_{L^{\infty}\left(0, \gamma_{1}\right)} \int_{0}^{\gamma_{1}} e^{-t x} d x+e^{-t \gamma_{1}} \int_{\gamma_{1}}^{\gamma}|V(x)| d x \leqslant \frac{1}{t}\|V\|_{\infty}+e^{-t \gamma_{1}}\|V\|_{1},
\end{aligned}
$$

which yields (5.6). By (3.7), the S-matrix has the form

$$
S(\lambda)=I-2 \pi i \mathcal{A}(\lambda), \quad \mathcal{A}=\mathcal{A}_{0}-\mathcal{A}_{1} .
$$

Using (15.3), (15.6) we obtain

$$
\mathcal{A}(\lambda)=\mathcal{A}_{0}(\lambda)-\mathcal{A}_{1}(\lambda)=\frac{e^{i \frac{2 \pi}{3}+\frac{4}{3} t^{3}}}{8 t^{2}}\left[(V(0)+o(1))+O\left(t^{-a}\right)\right],
$$

which permits to get (5.7).

We are now ready to prove the main theorems.

Proof of Theorem 1.2. It follows from Lemma 5.1 and 4.1 that $S(\lambda), \lambda \in \mathbb{R}$ has an analytic extension from $\mathbb{R}$ into the upper half-plane $\mathbb{C}_{+}$and satisfies

$$
|S(\lambda)| \leqslant C_{S} e^{\frac{4}{3}|\lambda|^{\frac{3}{2}}} \quad \forall \lambda \in \overline{\mathbb{C}}_{+},
$$

for some constant $C_{S}$. Invoking the identity (1.6) we obtain $D_{-}(\lambda-i 0)=S(\lambda) D_{+}(\lambda+i 0), \lambda \in$ $\mathbb{R}$. Thus it follows from Lemma 5.1, 4.1 and 2.3 that $D_{-}(\lambda), \lambda \in \mathbb{C}_{-}$has an analytic extension from $\overline{\mathbb{C}}_{-}$into the whole complex plane $\mathbb{C}$ and satisfies

$$
\begin{aligned}
& \left|D_{-}(\lambda)\right| \leqslant|S(\lambda)|\left|D_{+}(\lambda)\right| \leqslant C_{S} e^{\frac{4}{3}|\lambda|^{\frac{3}{2}}} \sup _{\lambda \in \mathbb{C}_{+}}\left|D_{+}(\lambda)\right| \quad \forall \lambda \in \overline{\mathbb{C}}_{+}, \\
& \sup _{\lambda \in \mathbb{C}_{ \pm}}\left|D_{ \pm}(\lambda)\right|<\infty,
\end{aligned}
$$

which together with (1.7) yields (1.13). Then, by (1.6), the S-matrix $S(\lambda), \lambda \in \mathbb{R}$ has an analytic extension into the whole upper half plane $\mathbb{C}_{+}$and a meromorphic extension into the whole lower half plane $\mathbb{C}_{-}$satisfying

$$
S(\lambda)=\frac{D_{-}(\lambda)}{D_{+}(\lambda)} \quad \forall \lambda \in \mathbb{C},
$$

where the functions $D_{ \pm}$do not vanish in $\overline{\mathbb{C}}_{ \pm}$. The zeros of $S(\lambda), \lambda \in \mathbb{C}_{+}$coincide with the zeros of $D_{-}$and the poles of $S(\lambda), \lambda \in \mathbb{C}_{-}$are precisely the zeros of $D_{+}$. 
Let, in addition, $V$ satisfy Condition $\mathrm{C}$ with $V(0) \neq 0$. Then asymptotics (5.7), (1.8) give

$$
D_{-}\left(t^{2} e^{i \frac{\pi}{3}}\right)=\frac{e^{i \frac{2 \pi}{3}+\frac{4}{3} t^{3}}}{8 t^{2}}(V(0)+o(1)) \quad \text { as } \quad t \rightarrow+\infty .
$$

Thus $D_{ \pm}$is an entire functions of order $\frac{3}{2}$ and type $\frac{4}{3}$.

Now we discuss Remarks after Theorem 1.2. By Theorem 1.2, the determinants $D_{ \pm}(\lambda), \lambda \in$ $\mathbb{C}_{ \pm}$extend to entire functions of order $\frac{3}{2}$ and type $\frac{4}{3}$. It is well known that in this case $D_{+}$has the Hadamard factorization (1.14) and (5.13) for some $p$, see p. 22 in [5]. Moreover, we have

$$
\frac{D_{+}^{\prime}(\lambda)}{D_{+}(\lambda)}=p+\sum_{n \geqslant 1} \frac{\lambda}{\lambda_{n}\left(\lambda-\lambda_{n}\right)}
$$

where the constant $p=\frac{D_{+}^{\prime}(0)}{D_{+}(0)}$ and the series converges absolutely and uniformly on any compact set of $\mathbb{C} \backslash\left\{\lambda_{n}, n \geqslant 1\right\}$. Recall that if an entire function $F$ has order $m>0$ and has zeros $z_{n}, n \geqslant 1$, then (see p. 17 in [5])

$$
\sum_{z_{n} \neq 0, n \geqslant 1}\left|z_{n}\right|^{-m_{1}}<\infty, \quad \forall m_{1}>m
$$

Using (1.14), (15.13) and differentiating $S(\lambda)=\frac{\bar{D}_{+}(\lambda+i 0)}{D_{+}(\lambda+i 0)}=e^{-2 \pi i \phi_{s c}(\lambda)}, \lambda \in \mathbb{R}$, we obtain

$$
S(\lambda)\left(-2 \pi i \phi_{s c}^{\prime}(\lambda)\right)=-S(\lambda)\left(\frac{D_{+}^{\prime}(\lambda+i 0)}{D_{+}(\lambda+i 0)}-\frac{\bar{D}_{+}^{\prime}(\lambda+i 0)}{\bar{D}_{+}(\lambda+i 0)}\right), \quad \lambda \in \mathbb{R}
$$

which yields

$$
2 \pi i \phi_{s c}^{\prime}(\lambda)=p-\bar{p}+\lambda \sum_{n \geqslant 1}\left(\frac{1}{\lambda_{n}\left(\lambda-\lambda_{n}\right)}-\frac{1}{\bar{\lambda}_{n}\left(\lambda-\bar{\lambda}_{n}\right)}\right), \quad \forall \lambda \in \mathbb{R},
$$

and then $2 \pi i \phi_{s c}^{\prime}(0)=p-\bar{p}$. Thus we obtain (1.19).

Finally, we prove Corollary 1.3 and 1.4 .

Proof of Corollary 1.3. Under the Condition V Theorem 1.2 gives that the determinant $D_{+}(\lambda), \lambda \in \mathbb{C}_{+}$extends to an entire function and satisfies (1.13), which yields the standard upper bound (1.16) (see page 16 in [5]).

Let $V$ satisfy the Condition $\mathrm{C}$ and $V(0) \neq 0$. Then by Theorem $\left[1.2\right.$, determinant $D_{+}(\lambda), \lambda \in$ $\mathbb{C}_{+}$is an entire function of order $\frac{3}{2}$ and type $\frac{4}{3}$. Since the order of $D_{+}$is not integer, (1.16) follows from the Lindelöf Theorem [36]. We recall this theorem.

Lindelöf Theorem (1903). Let $f$ be an entire function of order $\beta$, which is not an integer.

Then i) $f$ is of zero type iff $\mathcal{N}(r, f)=o\left(r^{\beta}\right)$,

ii) $f$ is of finite type iff $\mathcal{N}(r, f)=O\left(r^{\beta}\right)$.

Moreover, since the type of $D_{+}$is different from zero, $\mathcal{N}\left(r, D_{+}\right)$is not $o\left(r^{3 / 2}\right)$. This is (1.17).

Proof of Corollary 1.4. Due to (3.1) we have $\operatorname{Tr}\left(R(\lambda)-R_{0}(\lambda)\right)=\frac{D_{+}^{\prime}(\lambda)}{D_{+}^{\prime}(\lambda)}$ for each $\lambda \in \mathbb{C}_{+}$ and adding (5.13) we obtain (1.18). 
Proposition 5.3. Let $V$ satisfy Condition $\mathrm{V}$. Then for any $f \in C_{0}^{\infty}(\mathbb{R})$ the following identity

$$
\operatorname{Tr}\left(f(H)-f\left(H_{0}\right)\right)=-\frac{1}{\pi} \int_{\mathbb{R}} f(t)\left(\phi_{s c}^{\prime}(0)+\frac{\lambda}{\pi} \operatorname{Im} \sum_{n \geqslant 1} \frac{1}{\lambda_{n}\left(t-\lambda_{n}\right)}\right) d t,
$$

holds true, furthermore,

$$
\phi_{s c}^{(m)}(0)=\frac{(m-1) !}{\pi} \operatorname{Im} \sum_{n \geqslant 1} \frac{1}{\lambda_{n}^{m}}
$$

where the first series converges absolutely and uniformly on any compact set of $\mathbb{C} \backslash\left\{\lambda_{n}, n \geqslant 1\right\}$.

Proof. Due to (2.27) we obtain $R(\lambda)-R_{0}(\lambda) \in \mathcal{B}_{1}$ for each $\operatorname{Im} \lambda \neq 0$. Then the Krein formula [35] for the operators $H, H_{0}$ and for any $f \in C_{0}^{\infty}(\mathbb{R})$ gives

$$
\operatorname{Tr}\left(f(H)-f\left(H_{0}\right)\right)=-\int_{\mathbb{R}} f(t) \phi_{s c}^{\prime}(t) d t
$$

and substituting (1.19) into (5.18) we obtain (5.16).

Due to (1.19) we have the identity

$$
\phi_{s c}^{\prime}(\lambda)=\phi_{s c}^{\prime}(0)+\frac{1}{\pi} \operatorname{Im} \sum_{n \geqslant 1}\left(\frac{1}{\lambda_{n}-\lambda}-\frac{1}{\lambda_{n}}\right), \quad \forall \lambda \in \mathbb{C},
$$

uniformly on any compact subset of $\mathbb{C} \backslash\left\{\lambda_{1}, \lambda_{2}, \lambda_{3}, \cdots\right\}$. Differentiating $\phi_{s c}^{\prime}(\lambda)$ we then arrive at (5.17).

Proof of Theorem 1.5. There are results about inverse problems for perturbed Stark operators $H=H_{0}+V$ on $L^{2}(\mathbb{R})$. For example, Kachalov and Kurylev [24] consider inverse scattering problem, when the potential $V$ satisfies

$$
\int_{\mathbb{R}}(1+|x|)^{4}|V(x)| d x<\infty .
$$

They prove the recovering problem using the Gelfand-Levitan equation: for given S-matrix $S(\lambda)$ to determine a potential $V$, which satisfies (5.20) (Theorem 1 and 2 in [24]).

Let $V$ satisfy Condition V. Then due to Theorem (1.6) and (1.14) the S-matrix $S(\lambda)$ is given by

$$
S(\lambda)=S(0) S_{1}(\lambda), \quad S_{1}(\lambda)=e^{-2 i \lambda \phi_{s c}^{\prime}(0)} \lim _{r \rightarrow+\infty} \prod_{\left|\lambda_{n}\right| \leqslant r}\left(\frac{1-\frac{\lambda}{\bar{\lambda}_{n}}}{1-\frac{\lambda}{\lambda_{n}}}\right) e^{\frac{\lambda}{\bar{\lambda}_{n}}-\frac{\lambda}{\lambda_{n}}}, \quad \lambda \in \mathbb{C},
$$

uniformly on any compact subset of $\mathbb{C}$, where $S(0)=\frac{\bar{D}_{+}(0)}{D_{+}(0)}=e^{-2 i \phi_{s c}(0)}$. Asymptotics (3.11) implies $S(\lambda) \rightarrow 1$ as $\lambda \rightarrow \pm \infty$. Then we deduce that there exists a following limit:

$$
S(0)=S(\lambda) \bar{S}_{1}(\lambda)=\lim _{\lambda \rightarrow-\infty} \bar{S}_{1}(\lambda)
$$

Thus due to (5.21) and (5.22) the S-matrix is expressed in terms of resonances only.

Now we consider two perturbed Stark operators $H_{j}=H_{0}+V_{j}, j=1,2$ on $L^{2}(\mathbb{R})$, where the potential $V_{j}$ satisfies Condition V. We denote the S-matrix for $H_{0}, H_{j}$ by $S_{j}(\lambda)$. Assume that $H_{1}$ and $H_{2}$ have the same resonances. Then by the above results, the S-matrices for $H_{1}$ and $H_{2}$ coincide, i.e., $S_{1}=S_{2}$. After this the Kachalov and Kurylev results [24] give $V_{1}=V_{2}$. 


\section{Appendix}

Introduce the Fourier transformation

$$
\widehat{f}(t)=\frac{1}{\sqrt{2 \pi}} \int_{\mathbb{R}} f(x) e^{-i x t} d x, \quad t \in \mathbb{R} .
$$

Lemma 6.1. Let $V$ satisfy conditions in Theorem 1.1 and $\lambda \in \overline{\mathbb{C}}_{+}$. Then the following identity and asymptotics

$$
\begin{gathered}
\operatorname{Tr} Y_{0}(\lambda)=\frac{e^{i \frac{\pi}{4}}}{(4 \pi)^{1 / 2}} \int_{\mathbb{R}} V(x) d x \int_{0}^{\infty} e^{-i t^{3} / 12} e^{i t(\lambda-x)} \frac{d t}{t^{1 / 2}}=\frac{e^{i \frac{\pi}{4}}}{\sqrt{2}} \int_{0}^{\infty} e^{i t \lambda-\frac{i}{12} t^{3}} \widehat{V}(t) \frac{d t}{t^{1 / 2}}, \\
\operatorname{Tr} Y_{0}(\lambda)=i \frac{V_{0}}{2 \sqrt{\lambda}}+\frac{O(1)}{\lambda} \quad \text { as }|\lambda| \rightarrow \infty,
\end{gathered}
$$

hold true, uniformly with respect to $\arg \lambda \in[0, \pi]$.

Proof. We show (6.2). In view of (2.39) we have $Y_{0}(\lambda) \in \mathcal{B}_{1}$ for all $\lambda \in \mathbb{C}_{+}$. The identity (2.11) for $\lambda \in \mathbb{C}_{+}$with $c:=\frac{e^{i \frac{\pi}{4}}}{\sqrt{2}}$ gives

$\operatorname{Tr} Y_{0}(\lambda)=\int_{\mathbb{R}} V(x) R_{0}(x, x, \lambda) d x=\frac{c}{\sqrt{2 \pi}} \int_{\mathbb{R}} V(x) d x \int_{0}^{\infty} e^{i t(\lambda-x)-i \frac{t^{3}}{12}} \frac{d t}{t^{\frac{1}{2}}}=c \int_{0}^{\infty} e^{i t \lambda-\frac{i}{12} t^{3}} \widehat{V}(t) \frac{d t}{t^{\frac{1}{2}}}$.

We characterize the properties of $V$ in terms of its Fourier transform $\widehat{V}$ :

$$
\begin{aligned}
& \widehat{V},(\widehat{V})^{\prime},(\widehat{V})^{\prime \prime} \in L^{\infty}(\mathbb{R}) \cap L^{2}(\mathbb{R}), \\
& \widehat{V}(t)=\frac{V(0)-e^{-i t \gamma} V(\gamma)}{i t \sqrt{2 \pi}}+\frac{1}{i t \sqrt{2 \pi}} \int_{0}^{\gamma} e^{-i t x} V^{\prime}(x) d x=\frac{O(1)}{t} \quad \text { as } t \rightarrow \pm \infty .
\end{aligned}
$$

Let us show (6.3). Define cut-off functions $w_{0}, w_{1} \in C^{\infty}\left(\mathbb{R}_{+}\right)$by

$$
w_{0}(t)=\left\{\begin{array}{ll}
1 & t \in[0,1] \\
0 & t>2
\end{array}, \quad w_{1}=1-w_{0} .\right.
$$

Thus (6.2) yields the decomposition for $\lambda \in \overline{\mathbb{C}}_{+}$:

$$
\begin{aligned}
& \operatorname{Tr} Y_{0}(\lambda)=c \int_{0}^{\infty} e^{i t \lambda-\frac{i}{12} t^{3}} \widehat{V}(t) \frac{d t}{t^{1 / 2}}=c I_{0}(\lambda)+c I_{1}(\lambda), \quad c=\frac{e^{i \pi / 4}}{\sqrt{2}}, \\
& \text { where } I_{j}=\int_{0}^{\infty} w_{j}(t) e^{i \phi(t)} \widehat{V}(t) \frac{d t}{t^{1 / 2}}, \quad j=0,1, \quad \phi(t)=t \lambda-\frac{t^{3}}{12} .
\end{aligned}
$$

Let us first consider $I_{0}$. Recall that $\widehat{V}(t)$ and has the decomposition:

$$
e^{-\frac{i}{12} t^{3}} \widehat{V}(t)=\widehat{V}(0)+t \beta(t) \quad \text { as } t \rightarrow 0
$$

where $\widehat{V}(0)=\frac{V_{0}}{\sqrt{2 \pi}}$ and the function $\beta$ is entire. Substituting this asymptotics into $I_{0}$ we obtain

$$
I_{0}=\int_{0}^{2} w_{0}(t) e^{i t \lambda-\frac{i}{12} t^{3}} \widehat{V}(t) \frac{d t}{t^{1 / 2}}=I_{01}+I_{02}, \quad I_{01}=\widehat{V}(0) \int_{0}^{2} w_{0}(t) e^{i t \lambda} \frac{d t}{t^{1 / 2}} .
$$

The stationary phase method gives

$$
I_{01}=\frac{V_{0}}{\sqrt{2 \pi}} \int_{0}^{2} w_{0}(t) e^{i t \lambda} \frac{d t}{t^{1 / 2}}=\frac{e^{i \pi / 4} V_{0}}{\sqrt{2 \pi}} \sqrt{\frac{\pi}{\lambda}}+\frac{O(1)}{\lambda^{\frac{3}{2}}}=\frac{c V_{0}}{\sqrt{\lambda}}+\frac{O(1)}{\lambda^{\frac{3}{2}}},
$$


and an integration by parts further implies

$$
I_{02}=\int_{0}^{2} w_{0} e^{i t \lambda}\left(e^{-\frac{i}{12} t^{3}} \widehat{V}(t)-\widehat{V}(0)\right) \frac{d t}{t^{1 / 2}}=\frac{O(1)}{\lambda} .
$$

Thus, if we assume that $I_{1}=O(1 / \lambda)$, then (6.7) and (6.8) yield (6.3). Hence, it remains to show that $I_{1}=O(1 / \lambda)$. To this end we distinguish three cases.

- First, let $\lambda=\mu+i \nu, \nu \geqslant K|\mu|$ for some $K>0$ and let $\nu \rightarrow \infty$. We have

$$
\left|I_{1}\right| \leqslant \int_{1}^{\infty} e^{-t \nu}|\widehat{V}(t)| d t \mid \leqslant\|\widehat{V}\|_{\infty} \int_{1}^{\infty} e^{-t \nu} d t=\frac{\|\widehat{V}\|_{\infty}}{\nu} e^{-\nu}
$$

- Second, let $\operatorname{Re} \lambda \rightarrow-\infty$. We put $f=w_{1}(1) \widehat{V}(t) / t^{1 / 2}$ and here $\phi^{\prime}(t)=\lambda-\frac{t^{2}}{4}$. Then due to (6.4) we have

$$
I_{1}=\int_{1}^{\infty} e^{i \phi(t)} f(t) d t=\int_{1}^{\infty} \frac{f(t)}{i \phi^{\prime}(t)} d e^{i \phi(t)}=i \int_{1}^{\infty} e^{i \phi(t)}\left(\frac{f(t)}{\phi^{\prime}(t)}\right)^{\prime} d t=\frac{O(1)}{\lambda}
$$

since (6.4) yields $f^{\prime}, f=O\left(t^{-3 / 2}\right), t \rightarrow \infty$ and we have the following estimate

$$
\left|\left(\frac{f(t)}{i\left(\lambda-\frac{t^{2}}{4}\right)}\right)^{\prime}\right| \leqslant \frac{C}{|\lambda| t^{\frac{3}{2}}} \quad \forall t \geqslant 1,
$$

for some constant $C$

- Third, let $\lambda=\frac{k^{2}+i \nu}{4} \in \overline{\mathbb{C}}_{+}, k \rightarrow+\infty$ and $K k^{2} \geqslant \nu$. Define the cut-off functions $g_{0}, g_{1} \in C_{0}^{\infty}(\mathbb{R})$ by

$$
g(s)=\left\{\begin{array}{ll}
1 & s \in(-1 / 2,1 / 2) \\
0 & s \in \mathbb{R}_{+} \backslash(-1,1)
\end{array}, \quad g_{1}=1-g(t-k) .\right.
$$

Then we have

$$
I_{1}=\int_{1}^{\infty} e^{i t \lambda-\frac{i}{12} t^{3}} f(t) d t=I_{11}+I_{12}, \quad I_{11}=\int_{1}^{\infty} e^{i t \lambda-\frac{i}{12} t^{3}} g(t-k) f(t) d t, \quad f=\widehat{V}(t) \frac{w_{1}(t)}{t^{\frac{1}{2}}} .
$$

Let $F(t)=g_{1}(t) f(t)$. Similar to (6.10) we obtain

$$
\begin{aligned}
I_{12}=\int_{1}^{\infty} e^{i \phi(t)} F(t) d t= & \int_{1}^{\infty} \frac{F(t)}{i \phi^{\prime}(t)} d e^{i \phi(t)}=-\int_{1}^{\infty} e^{i \phi(t)}\left(\frac{F(t)}{i \phi^{\prime}(t)}\right)^{\prime} d t \\
& =-\int_{1}^{\infty} e^{i \phi(t)}\left(\frac{1}{\phi^{\prime}(t)}\left(\frac{F(t)}{\phi^{\prime}(t)}\right)^{\prime}\right)^{\prime} d t=\frac{O(1)}{|\lambda|}
\end{aligned}
$$

since due to (6.4) we have

$$
\left|\left(\frac{1}{\phi^{\prime}(t)}\left(\frac{F(t)}{\phi^{\prime}(t)}\right)^{\prime}\right)^{\prime}\right| \leqslant \frac{C}{|\lambda| t^{\frac{3}{2}}} \quad \forall t \geqslant 1 .
$$

Let us now consider the main term $I_{11}$. We have for $\lambda=\frac{k^{2}+i \nu}{4}$ and $t=k+s$ :

$$
\begin{array}{r}
t \lambda-\frac{i}{12} t^{3}=\frac{1}{12}\left(3(k+s)\left(k^{2}+i \nu\right)-(k+s)^{3}\right)=\frac{1}{12}\left(3(k+s) k^{2}+i 3 t \nu-(k+s)^{3}\right) \\
=\frac{1}{12}\left(2 k^{3}+i 3 t \nu-3 s^{2} k-s^{3}\right)=\frac{k^{3}}{6}+i \nu \frac{k+s}{4}+-\frac{k}{4} s^{2}-\frac{s^{3}}{12} .
\end{array}
$$


Define $\psi(s, \lambda)=e^{-i \frac{s^{3}}{12}} e^{-\frac{\nu}{4}(k+s)} g(s) f(k+s)$ and note that $f(k+s)=O\left(k^{-\frac{3}{2}}\right)$ as $k \rightarrow \infty$, uniformly in $s \in[-1,1]$. Due to (6.4) the stationary phase method gives

$$
I_{11}=\int_{k-1}^{k+1} e^{i t \lambda-\frac{i}{12} t^{3}} g(t-k) f(t) d t=e^{\frac{i}{6} k^{3}} \int_{-1}^{1} e^{-\frac{i}{4} k s^{2}} \psi(s, \lambda) d s=\frac{O(1)}{k^{2}}=\frac{O(1)}{|\lambda|} .
$$

Combining (6.9)-(6.12) we obtain $I_{1}=O(1 / \lambda)$ and (6.3) .

Acknowledgments. Various parts of this paper were written at the Mathematical Institute of Potsdam University. E.K. is grateful to the institute for the kind hospitality. He is also grateful to Alexei Alexandrov (St. Petersburg), Vladimir Peller (Michigan) and Michail Sodin (Tel-Aviv) for stimulating discussions and useful comments about entire functions. Moreover, he is also grateful to Oliver Matte (Aarhus) for reading the manuscript and useful remarks. The author would like to thank the referee for useful comments. His study was supported by the RSF grant No. 15-11-30007.

\section{REFERENCES}

[1] Agler, J.; Froese, R. Existence of Stark ladder resonances. Commun. Math. Phys. 100(1985), $161-171$.

[2] Aleksandrov, A. B. Norm of the Hilbert transformation in a space of Hölder functions, Functional Analysis and Its Applications, 9(1975), no 2, 94-96.

[3] Avron, J.; Herbst, I. Spectral and scattering theory of Schrödinger operators related to the Stark effect, Commun. Math. Phys. 52(1977), 239-254.

[4] Barreto, A. Remarks on the distribution of resonances in odd dimensional Euclidean scattering. Asymptot. Anal. 27 (2001), no. 2, 161-170.

[5] Boas, R. Jr. Entire functions. Academic Press Inc., New York, 1954.

[6] Brown, B.; Knowles, I.; Weikard, R. On the inverse resonance problem, J. London Math. Soc. 68 (2003), no. 2, 383-401.

[7] Calogero, F.; Degasperis, A. Inverse spectral problem for the one-dimensional Schrödinger equation with an additional linear potential. Lettere Al Nuovo Cimento 23(1978), no. 4, 143-149.

[8] Cycon, H.; Froese, R.; Kirsch, W.; Simon, B, Schrödinger operators with applications to quantum mechanics and global geometry. Springer, 1987.

[9] Deift, P.; Killip, R. On the absolutely continuous spectrum of one-dimensional Schrödinger operators with square summable potentials, Commun. Math. Phys. 203 (1999), 341-347.

[10] Faddeev, L. D. On a model of Friedrichs in the theory of perturbations of the continuous spectrum. (Russian) Trudy Mat. Inst. Steklov 73 (1964), 292-313.

[11] Faddeev, L.; Zakharov, V. The Korteweg-de Vries equation is a a completely integrable Hamiltonian system. Functional Analysis and Its Applications, 5(1971), no 4, 280-287.

[12] Firsova, N. Resonances of the perturbed Hill operator with exponentially decreasing extrinsic potential. Mathematical Notes, 36(1984), no 5, 854-861.

[13] Froese, R. Asymptotic distribution of resonances in one dimension. J. Diff. Eq. 137 (1997), no. 2, $251-272$.

[14] Herbst, I. Unitary equivalence of Stark Hamiltonians, Math. Z. 155 (1977), 55-70.

[15] Herbst, I. Dilation analyticity in constant electric field, I, The two body problem, Commun. Math. Phys. 64 (1979), 279-298.

[16] Herbst, I. W.; Howland, J. S. The Stark ladder and other one-dimensional external field problems, Commun. Math. Phys. 80(1981), 23-42.

[17] Gohberg, I.; Krein, M. Introduction to the theory of linear nonselfadjoint operators. Translated from the Russian, Translations of Mathematical Monographs, Vol. 18 AMS, Providence, R.I. 1969

[18] Graffi, S.; Harrell, E. Inverse scattering for the one-dimensional Stark effect and application to the cylindrical KdV equation, Annales de l'institut Henri Poincaré (A) Physique theorique, 36 (1082), 4158.

[19] Grecchi, V.; Sacchetti, A. Lifetime of the Wannier-Stark resonances and perturbation theory. Commun. Math. Physics 185 (1997), no. 2, 359-378.

[20] Its, A.; V. Sukhaviv, V. Riemann-Hibert approach for the inverse problem for the Stark operator on the line, preprint 2015. 
[21] Jensen, A. Asymptotic completeness for a new class of Stark effect Hamiltonians, Commun. Math. Phys. 107(1986), 21-28.

[22] Jensen, A. Commutator methods and asymptotic completeness for one-dimensional Stark effect Hamiltonians Schrödinger Operators, Aarhus 1985, Lecture Notes in Mathematics, Volume 1218 (1986), 151-166.

[23] Jensen, A. Perturbation results for Stark effect resonances. J. Reine Angew. Math 394 (1989), 168-179.

[24] Kachalov, A.; Kurylev, Y. The method of transformation operators in the inverse scattering problem. The one-dimensional Stark effect. Journal of Soviet Mathematics 57(1991), no. 3, 3111-3122.

[25] Kargaev, P.; Korotyaev, E. Inverse Problem for the Hill operator, the Direct Approach. Invent. Math. 129(1997), no. 3, 567-593.

[26] Koosis P. The logarithmic integral I, Cambridge Univ. Press, Cambridge, London, New York 1988.

[27] Korotyaev, E. On the theory of multiparticle scattering in an external electric field. (Russian) Mat. Sb. (N.S.) 132(174) (1987), no. 2, 182-201, translation in Math. USSR-Sb. 60(1988), no. 1, 177-196.

[28] Korotyaev, E. Inverse resonance scattering on the half line. Asymptot. Anal. 37 (2004), no. 3-4, 215-226.

[29] Korotyaev, E. Inverse resonance scattering on the real line. Inverse Problems 21(2005), no. 1, 325-341.

[30] Korotyaev, E. Resonance theory for perturbed Hill operator, Asymp. Anal. 74(2011), no. 3-4, 199-227.

[31] Korotyaev, E.; Pushnitski, A. Trace formulae and high energy asymptotics for the Stark operator. Comm. Partial Differential Equations 28 (2003), no. 3-4, 817-842.

[32] Korotyaev, E.; Schmidt, K. On the resonances and eigenvalues for a 1D half-crystal with localized impurity, J. Reine Angew. Math. 2012, Issue 670, 217-248.

[33] Kristensson, G. The one-dimensional inverse scattering problem for an increasing potential. Jour. Math. Phys. 27 (1986), no. 3, 804-815.

[34] Laptev, A.; Weidl, T., Sharp Lieb-Thiring inequalities in high dimensions, Acta Math., 184(2000), 87111.

[35] Krein, M. G. On perturbation determinants and the trace formula for unitary and selfadjoint operators, Soviet Math. Dokl. 3 (1962), 707-710.

[36] Lindelöf, E. Sur les functions entires d'odre entire, Ann., Sci., Ecole Norm. Sup. 31,22 (1903), 369-395.

[37] Lin, Y.; Qian, M.; Zhang, Q., Inverse scattering problem for one-dimensional Schrdinger operators related to the general Stark effect. Acta Mathematicae Applicatae Sinica 5 (1989), no. 2, 116-136.

[38] Liu, Y. Scattering and spectral theory for Stark Hamiltonians in one dimension. Mathematica Scandinavica 72 (1993), 265-297.

[39] Olver, F. W. J. Asymptotics and Special Functions, Academic Press, New York, 1974.

[40] Reed, M.; Simon, B. Methods of Modern Mathematical Physics, Vol. III: Scattering Theory, Academic Press, New York, 1979.

[41] Reed, M.; Simon, B. Methods of Modern Mathematical Physics, Vol.IV: Analysis of Operators, Academic Press, New York, 1978.

[42] Regge, T. Analytic properties of the scattering matrix, Nuovo Cimento, 8 (1958), no. 5, 671-679.

[43] Rejto, P. A.; Sinha, K. Absolute continuity for a 1-dimensional model of the Stark-Hamiltonian. Helv. Phys. Acta 49(1976), 389-413.

[44] Simon, B. Resonances in one dimension and Fredholm determinants, J. Funct. Anal. 178 (2000), no. 2, $396-420$.

[45] Simon, B. Trace ideals and their applications. Second edition. Mathematical Surveys and Monographs, 120. AMS, Providence, RI, 2005.

[46] Sjöstrand, J.; Zworski, M. Complex scaling and the distribution of scattering poles. J. Amer. Math. Soc. 4 (1991), no. 4, 729-769.

[47] Yajima, K. Spectral and scattering theory for Schrodinger operator with Stark effect, J. Fac. Sci. Univ. Tokyo. S. IA. 26(1979), 377-390.

[48] Yajima, K. Spectral and scattering theory for Schrodinger operators with Stark effect. II, J. Fac. Sci. Univ. Tokyo. S. IA. 28(1981), 1-15.

[49] Zworski, M. Sharp polynomial bounds on the number of scattering poles of radial potentials. J. Funct. Anal. 82 (1989), no. 2, 370-403.

[50] Zworski, M. Distribution of poles for scattering on the real line, J. Funct. Anal. 73(1987), 277-296.

[51] Zworski, M. SIAM, J. Math. Analysis, "A remark on isopolar potentials" 82(2002), no. 6, 1823-1826. 
Saint-Petersburg State University, Universitetskaya nab. 7/9, St. Petersburg, 199034, RUSSIA, KOROTYAEV@GMAIL.COM, E.KOROTYAEV@SPBU.RU 